\title{
Orbital circularisation of white dwarfs and the formation of gravitational radiation sources in star clusters containing an intermediate mass black hole
}

\author{
P. B. Ivanov ${ }^{1,2}$ and J. C. B. Papaloizou ${ }^{1}$ \\ ${ }^{1}$ Department of Applied Mathematics and Theoretical Physics, CMS, University of Cambridge, Wilberforce Road, \\ Cambridge, CB3 0WA, UK \\ e-mail: pbi20@damtp.cam.ac.uk \\ 2 Astro Space Center, P. N. Lebedev Physical Institute, Profsouyznaya St., 84/32 Moscow, Russia
}

Received 15 January 2007 / Accepted 31 August 2007

ABSTRACT

\begin{abstract}
Aims. We consider how tight binaries consisting of a super-massive black hole of mass $M=10^{3}-10^{4} M_{\odot}$ and a white dwarf in quasi-circular orbit can be formed in a globular cluster. We point out that a major fraction of white dwarfs tidally captured by the black hole may be destroyed by tidal inflation during ongoing tidal circularisation, and therefore the formation of tight binaries is inhibited. However some fraction may survive tidal circularisation through being spun up to high rotation rates. Then the rates of energy loss through gravitational wave emission induced by tidally excited pulsation modes and dissipation through non linear effects may compete with the rate of increase of pulsation energy due to dynamic tides. The semi-major axes of these white dwarfs are decreased by tidal interaction below a "critical" value where dynamic tides decrease in effectiveness because pulsation modes retain phase coherence between successive pericentre passages.

Methods. We estimate the rate of formation of such circularising white dwarfs within a simple framework, modelling them as $n=$ 1.5 polytropes and assuming that results obtained from the tidal theory for slow rotators can be extrapolated to fast rotators.

Results. We estimate the total capture rate as $\sim N \sim 2.5 \times 10^{-8} M_{4}^{1.3} r_{0.1}^{-2.1} \mathrm{yr}^{-1}$, where $M_{4}=M / 10^{4} M_{\odot}$ and $r_{0.1}$ is the radius of influence of the black hole expressed in units $0.1 \mathrm{pc}$. We find that the formation rate of tight pairs is approximately 10 times smaller than the total capture rate, for typical parameters of the problem. This result is used to estimate the probability of detection of gravitational waves coming from such tight binaries by LISA.

Conclusions. We conclude that LISA may detect such binaries provided that the fraction of globular clusters containing black holes in the mass range of interest is substantial and that the dispersion velocity of the cluster stars near the radius of influence of the black hole exceeds $\sim 20 \mathrm{~km} \mathrm{~s}^{-1}$.
\end{abstract}

Key words. black hole physics - gravitational waves - stellar dynamics - white dwarfs - galaxies: star clusters - stars: oscillations

\section{Introduction}

There are some observational indications and theoretical suggestions that favour the presence of black holes in the mass range $\sim 10^{2}-10^{4} M_{\odot}$ in the centres of globular clusters. The observational arguments supporting this hypothesis relate to kinematical phenomena observed in the centres of some globular clusters (e.g. Gebhardt et al. 2000; Gebhardt et al. 2002) and the presence of X-ray sources not associated with the central nuclei in certain galaxies (e.g. Fabbiano 1989; Matsumoto et al. 2001; Ghosh et al. 2006, and references therein). There are also some theoretical models of the formation of such systems (e.g. Miller \& Hamilton 2002). A review of the observational and theoretical aspects of this problem has been recently given by van der Marel (2004).

In this Paper we assume that there is a black hole of mass $M \sim 10^{3}-10^{4} M_{\odot}$ in a star cluster and estimate the rate of capture of white dwarfs of mass $m$ by the black hole. The capture rate is always determined by the interplay of two processes. These are the effect of distant two body gravitational encounters changing the orbital angular momenta of the stars and an interaction associated with the presence of the black hole which removes the orbital energy of a star and is effective only when the orbital angular momentum is sufficiently small. This type of interaction may result either through tidal interactions or by the emission of gravitational waves induced by the stellar orbital motion.

The efficiency of tidal interactions is determined by the ratio of orbital pericentre distance to the tidal radius - the latter being the distance from the black hole below which significant disruption of the star through mass loss induced by tides occurs. On the other hand, the efficiency of orbital energy loss due to gravitational wave emission is determined by the ratio of the orbital pericentre distance to the gravitational radius of the black hole.

Since the tidal radii corresponding to white dwarfs for the range of black hole masses considered here are larger than their gravitational radii, orbital energy is changed mainly through the action of dynamical tides. This is in contrast to the case of the more massive black holes residing in galactic centres where emission of gravitational waves is more effective for changing the orbital energy of white dwarfs (e.g. Ivanov 2002; Freitag 2003, and references therein).

Since the relative contribution of two body gravitational encounters to the orbital evolution decreases very sharply for small angular momenta, a star with sufficiently small angular momentum loses orbital energy through tidal interaction while the orbital angular momentum remains approximately unchanged. The 
latter occurs because the star cannot store a significant amount of angular momentum in comparison to the orbit. Accordingly, the orbital eccentricity decreases during this process which will be referred to as orbital circularisation.

As a result of this process a tight quasi-circular orbit around a black hole may be formed. A white dwarf on such an orbit can emit gravitational radiation in the frequency band of order of $10^{-2} \mathrm{~Hz}$ which is the most favoured for the planned LISA space borne gravitational wave antenna. In principal this is able to detect gravitational waves with dimensionless amplitude as small as $10^{-24}$, for an observational time of one year. That means that the presence of such a white dwarf can be detected from distances of order of $\sim 10^{3} \mathrm{Mpc}$. Taking into account the fact that globular clusters form a very abundant population of cosmic objects such systems may contribute significantly to the budget of sources of gravitational radiation available for LISA.

There is one principal obstacle inhibiting formation of a binary pair consisting of a black hole of mass $M$ and a white dwarf of mass $m$ in a tight orbit around it. Because it is produced through tidal interaction, its semi-major axis will be close to the tidal radius. There the ratio of orbital binding energy to gravitational energy of the white dwarf is of order of $(M / m)^{2 / 3} \gg 1$. Thus, for such an orbit to be produced, an amount of energy far exceeding the internal binding energy of the white dwarf must be removed from it. When tides are effective, orbital energy is transferred to pulsation modes and thence to the internal energy of the star. Therefore, without an effective energy loss mechanism, the white dwarf could be easily unbound and thus destroyed by the tidal input of energy or tidal inflation.

Here we propose and discuss such a mechanism, which may, in principle, allow unbinding due to tidal energy input to be circumvented for a range of orbital parameters of the star. The operation of this mechanism depends on the interplay of several factors influencing the orbital evolution of star which we introduce below.

The character of orbital evolution under the influence of tides is mainly determined by three factors: 1) the time scale for decay of the stellar pulsations excited by tidal interaction, 2) the orbital parameters of the star, and 3 ) the rotation of the star.

Let us first consider the decay of pulsations in a non-rotating star. In this paper we assume that decay of stellar pulsations is caused both by the emission of gravitational waves that occurs because of the time-dependent density perturbations associated with them, and also by the dissipation of pulsation energy leading to its conversion into internal energy of the star. The latter decay channel is assumed to result through non-linear effects. Since its properties are very poorly understood at the present time, we shall consider the corresponding dissipation time scale to be a free parameter. But we shall assume that it is larger than the orbital period of the star. However, this is not an essential assumption for orbital evolution at sufficiently large semi-major axes, see below.

Initially the stellar orbit will be highly eccentric with a tidal interaction that excites stellar pulsations occurring impulsively at pericentre passage (e.g. Lai 1997; Ivanov \& Papaloizou 2004, hereafter IP). For long decay time scales, pulsations will always be present in the star, and as a result of every periastron passage, a new perturbation excited by tides is added.

When orbital semi-major axis is sufficiently large or the orbital period sufficiently long, it has been established that tidally induced changes to it cause the phase correlation between preexisting pulsations and freshly excited ones to be lost. Then, both the energy content of excited pulsation modes and the orbital energy of the star evolve in a stochastic manner (e.g. Kochanek 1992; Kosovichev \& Novikov 1992; Mardling 1995). Under this evolution, the mode energy and the orbital binding energy of the star grow on average, with a part of the mode energy being transferred to the internal energy of the star. Therefore the stochastic exchange of energy between the orbit and stellar pulsations leads to a decrease of the orbital semimajor axis and period as well as an increase of the internal energy of the star.

However, once the orbital period is sufficiently short, or the semi-major axis is below a critical value, pulsation modes can maintain phase coherence between successive pericentre passages, stochastic evolution ceases and dynamic tides are expected to become less efficient. At this point, the tidal evolution rate is determined by the natural decay timescales of the pulsation modes. A steady pulsation energy typical of that induced through one pericentre passage may be maintained, rather than the growth that occurs through the cumulative effect of mode energy inputs proceeding over many pericentre passages when the semi-major axis is large.

An important issue is whether the internal energy added to the star during the phase of stochastic evolution is enough to cause its destruction. For a non-rotating star the internal energy obtained by the star when the critical semi-major axis is reached is larger than the stellar gravitational energy. Therefore, such a star could be disrupted by tidal heating before the critical semimajor axis is reached.

However, during orbital circularisation orbital angular momentum is also transferred to the star until an equilibrium rotation rate is attained. This requires a rotation rate corresponding to corotation at periastron or faster. Thus the star can be spun up to high rotation rates. When measured in terms of the amount of energy input per periastron passage, the efficiency of tidal interaction is minimised for such a rotating star. See for example Fig. 8 of Lai (1997) and also IP. Accordingly the time scale of circularisation is increased.

In this situation, when dissipation of the mode energy as a result of non linear effects is not effective, the time scale for transmitting orbital energy to the pulsation modes may be larger than the time scale for removal of the pulsation energy through gravitational waves emitted because of the time-dependent perturbation of the star. In this situation the white dwarf may reach the critical semi-major axis without internal dissipation causing tidal inflation because of cooling by emission of gravitational waves. In the opposite limit of effective mode dissipation and internal heating, the rapidly rotating white dwarf may attain the critical semi-major axis through the emission of gravitational waves induced by orbital motion rather than through tidal interaction.

Thus taking into account the reduction in effectiveness of the tidal energy transfer brought about by the effect of stellar rotation, we find that for sufficiently large orbital angular momenta the semi-major axis can be decreased below the critical one without significant heating of the star.

After the critical semi-major axis is reached, again, because of the reduced efficiency of the tidal interaction, orbital evolution is governed by the emission of gravitational waves determined by the orbital motion of the white dwarf. This process can further reduce the orbital semi-major axis and lead to formation of a tight quasi-circular orbit.

We formulate the criterion for white dwarf "survival" treating dynamic tides within the framework of the simplest possible model of tidal interactions. We assume that the internal structure of a white dwarf is the same as that of a $n=1.5$ polytrope. We also assume that the results obtained from the theory of dynamic tides in slowly rotating stars can be extrapolated to high rotation 
rates for the purpose of making approximate estimates. Based on these assumptions we estimate the formation rate of tight pairs which turns out to be an order of magnitude smaller than the total rate of tidal capture, for typical parameters of the problem. This allows us to obtain an estimate of probability of detection of such sources of gravitational waves by LISA. We conclude that LISA could, in principal, detect such a source provided that there is a significant fraction of globular clusters containing black holes with masses $\sim 10^{3}-10^{4} M_{\odot}$, and with stellar velocity dispersions in their innermost regions exceeding $\sim 20 \mathrm{~km} \mathrm{~s}^{-1}$.

There are a number of different possibilities and branchings associated with the orbital evolution subsequent to tidal capture. Each of these requires consideration of several physical processes and is described by a number of algebraic expressions. In order to clarify the situation, we provide a more transparent summary of the proposed paths to a circularised orbit with disruption of the star avoided. In Fig. 1 we give a diagrammatic illustration of the possible orbital evolutionary paths that can be taken by a white dwarf subsequent to tidal capture by an intermediate mass black hole. Initially, impulsive energy and angular momentum exchanges between the orbit and star that occur every pericentre passage spin it up and excite modes of oscillation. As a result the rate of tidal evolution of the orbit decreases such that gravitational radiation can become more important. This may also be important for damping the oscillation modes. If the tidal capture occurs at sufficiently large pericentre distance, the importance of gravitational radiation during the orbital and pulsation mode evolution may allow the star to survive the regime of impulsive energy input at pericentre passage and become circularised without disruption and be a potential LISA source.

The plan of the paper, in which the above phenomena are considered in more detail, is as follows. In Sect. 2 we describe the model of the stellar cluster we use and the effect of distant two body encounters. In Sect. 3 we go on to discuss the tidal interaction of a white dwarf with the central black hole and pulsation mode excitation through dynamic tides. Then in Sect. 4 we consider the way in which gravitational wave emission/non linear effects may lead to pulsation mode amplitude limitation. In Sect. 5 we go on to explore the conditions for safe circularisation or tidal disruption of the white dwarf. In Sect. 6 we discuss the rate of tidal capture of white dwarfs by the black hole and estimate the fraction that can circularise safely, with technical details being relegated to an Appendix. In Sect. 7 we estimate the probability of detection of this source of gravitational radiation. Finally in Sect. 8 we discuss our results.

\section{The stellar system}

\subsection{Density distribution}

In order to discuss the rate of orbital circularisation for white dwarfs it is first necessary to consider the properties of the star cluster in which they are situated. This is because dynamical interaction with the stars in the system is responsible for the production of orbits for which tidal interaction with the central black hole becomes significant.

The radius of influence of the central black hole, $r_{a}$, is defined as the radius within which the total mass of the stars is the same as the mass of the central black hole: $M_{\mathrm{st}}\left(r_{a}\right)=M$. As has been noted by many authors, when $r<r_{a}$, a "cusp" in the stellar distribution of stars is formed with a density distribution such that

$\rho \propto r^{-(3 / 2+p)}$,

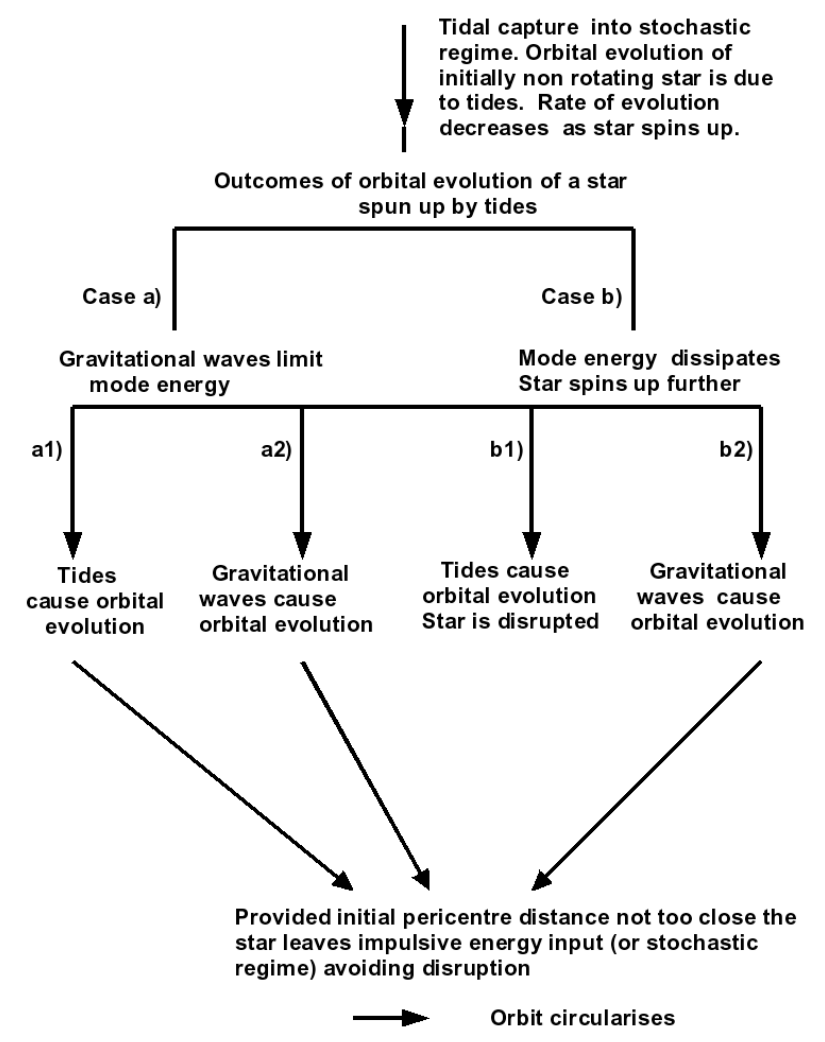

Fig. 1. A schematic illustration of the possible orbital evolutionary paths of a white dwarf that is tidally captured by an intermediate mass black hole. The star is initially scattered into a highly elongated trajectory that evolves due to impulsive energy and angular momentum exchanges between the star and orbit that occur every pericentre passage, the pericentre distance remaining very nearly fixed. As a result of these, the internal energy and angular momentum increase. However, this process is slowed as the star spins up and under favourable conditions gravitational radiation can become important so that it controls the orbital evolution and/or damps the excited pulsation modes. The latter case a) leading to cases a1) and a2) occurs where other non linear processes are ineffective at dissipating the pulsations. Case b) leading to cases b1) and b2) occurs when such processes are more effective. For both cases a) and b) the star may survive the orbital evolution if the tidal capture occurs at sufficiently large pericentre distance.

where the parameter $p$ is typically small. Two values that have been suggested through theoretical considerations are $p=0$ (Young 1980) and $p=1 / 4$ (Gurevich 1964; Bahcall \& Wolf 1976). Recently simulations by Baumgardt et al. (2004a,b) have indicated that for the case of globular clusters having a stellar population with a single mass, $p \approx 1 / 4$, while for systems with a realistic multi-mass stellar population, $p \approx 0.05$, with $p \approx 1 / 4$ for the most massive stars.

In this paper we make only approximate estimates of the tidal circularisation rate. Thus for simplicity, we have adopted $p=0$ for all components of the stellar system. We note that if a steeper cusp profile were to be adopted, the number of tidally captured stars at any particular time would increase by a numerical factor of order unity.

\subsection{Phase space distribution}

Making the usual assumption that the phase space distribution function for "typical" stars depends on the binding energy per unit mass only and the distribution of white dwarfs is the same as that of typical stars, it can be easily shown from (1) with $p=0$ 
that the distribution function for the white dwarfs over binding energy per unit mass and specific angular momentum, $N_{\mathrm{wd}}^{0}(E, J)$, is

$N_{\mathrm{wd}}^{0}(E, J)=2^{-3 / 2} \delta\left(\frac{M}{m r_{a}^{3}}\right)\left(G M r_{a}\right)^{-3 / 2} P_{\mathrm{orb}}(E) J$,

where $M$ and $m$ are the masses of the black hole and of a typical star, and we assume that $m \approx M_{\odot} . \delta \sim 0.1$ is the number fraction of white dwarfs in the cusp, $E$ and $J$ are, respectively, the binding energy per unit mass and the specific angular momentum of a star. We adopt the convention that the binding energy has positive values for the gravitationally bound stars considered in this paper.

$P_{\mathrm{orb}}(E)=\frac{\pi}{\sqrt{2}} \frac{G M}{E^{3 / 2}}$

is the orbital period of a star with binding energy per unit mass $E$.

\subsection{Loss cone}

The assumption of isotropy of the phase space distribution function is applicable only for estimates of quantities determined by the bulk of the stars, such as e.g. quantities characterising distant gravitational interactions, see Eq. (5) below. It becomes invalid for stars with sufficiently low orbital angular momentum which can either be tidally disrupted or directly captured by the black hole. The presence of the black hole thus leads to the formation of a "loss cone" such that Eq. (2) over-estimates the number of stars having low angular momenta. Assuming that stars with sufficiently small specific angular momenta $J<J_{\text {cone }}$ are absent, the presence of the loss cone may be easily accounted for by a correction factor (e.g. Lightman \& Shapiro 1977) such that the distribution function of the white dwarfs is modified to become

$N_{\mathrm{wd}}=\frac{\log \Theta}{\Lambda_{1}} N_{\mathrm{wd}}^{0}$

where $\Theta=J / J_{\text {cone }}, \Lambda_{1}=\ln \left(J_{\text {circ }}(E) / J_{\text {cone }}\right)$, and $J_{\text {circ }}$ is the specific angular momentum corresponding to a circular orbit with a given value of the binding energy per unit mass $E$. Typically, $\Lambda_{1} \sim 8$.

\subsection{Distant encounters}

A star orbiting around the black hole changes its energy and angular momentum due to distant gravitational interactions with other stars in the cusp. These result in a random walk in phase space. The stochastic change of the specific angular momentum per orbital period may be characterised by its dispersion, $\left(\Delta j_{2}\right)^{2}$, which can be written in the form (e.g., Ivanov 2002):

$\left(\Delta j_{2}\right)^{2}=\frac{29 \pi}{20} \Lambda_{2} q\left(G M r_{a}\right) \mathcal{E}^{-5 / 2}$,

where $q=m / M$ is the mass ratio, $\Lambda_{2} \approx \ln \left(0.5 q^{-1}\right)$ and we use the dimensionless energy variable $\mathcal{E}$ defined through $\mathcal{E}=$ $\operatorname{Er}_{a} /(G M){ }^{1}$

\footnotetext{
${ }^{1}$ In what follows it is convenient to represent different energy scales in dimensionless form. There are two natural units of energy per unit mass in our problem: the first associated the stellar cusp $-G M / r_{a}$ and the second associated with the internal structure of star itself $-G m / r_{\mathrm{wd}}$. For the dimensionless quantities expressed in terms of the first unit we will use the calligraphic style while the quantities expressed in terms of the second unit will be denoted by tilde.
}

\section{Tidal interaction of a white dwarf with the central black hole}

\subsection{White dwarf model}

In order to make order of magnitude estimates we use the simplest possible model for the white dwarf. We assume zero temperature so that the pressure is due to completely degenerate electrons. In this case the equation of state is baratropic and gravity or $g$ modes are absent. Furthermore we approximate the structure of the white dwarf as that of a $n=1.5$ polytrope. In addition to the mass $m$ and radius $R_{\mathrm{wd}}$ we introduce the two parameters $\mu=m / M_{\odot}$ and $\lambda=R_{\mathrm{wd}} /\left(0.01 R_{\odot}\right) \approx R_{\mathrm{wd}} /\left(7 \times 10^{8} \mathrm{~cm}\right)$. The effectiveness of tidal interactions depends significantly on the mean density of the white dwarf. To characterise low and high density cases we consider two sets of values for $\mu$ and $\lambda$. The "low density" case will be characterised by parameters appropriate for a "typical" white dwarf for which $\mu=0.6$ and $\lambda=1.4$, and for the "high density" case we use parameters corresponding to Sirius B for which $\mu \approx 1$ and $\lambda \approx 0.84$.

\subsection{Tidal perturbations and orbital circularisation}

For a baratropic star only fundamental $(f)$, pressure $(p)$ and inertial modes can be excited by tidal interaction. Since $p$-modes have large eigen-frequencies and inertial modes are significant only for rather large orbital angular momenta (Papaloizou \& Ivanov 2005), their respective contributions to the tidal exchange of energy and angular momentum are small compared to the contribution of the $f$-modes for the range of the orbital parameters of interest, so they are not considered further.

To make our estimates, we use the linear theory of tidal perturbations induced in a slowly rotating star and consider only the leading order quadrupole response. The assumption of slow rotation is certainly valid in the early stages of orbital evolution due to tides. In this regime orbital changes induced by distant gravitational interactions and tidal effects compete with each other. Then we may assume that the star is non-rotating when calculating the rate of tidal circularisation.

However, the star can be spun up because of the effect of tides during orbital circularisation, achieving a considerable rotation rate during the later stages. As we will see below this may have important implications for the outcome of the orbital evolution of the star. In particular, it may play a role in determining whether is it possible or not for the star to survive in a tight quasicircular orbit. Therefore, it is directly related to the problem of formation of sources of gravitational radiation. Unfortunately, a theory of tidal perturbations of a fast rotating star is practically undeveloped in the present time. Accordingly, we shall assume that the results obtained from the theory of slowly rotating stars can be extrapolated to the case of fast rotation for the purposes of making order of magnitude estimates.

During the early stages of tidal circularisation, the orbit of the star is highly eccentric. In this case tidal interactions are only important near pericentre. Because of this, in order to calculate the energy and angular momentum exchange between the orbit and the modes of pulsation of the star it is possible to consider it as undergoing a sequence of successive flybys of the black hole each of which produces an impulsive change. This formulation of the problem allows us to use the theory of tidal excitation and dissipation of the fundamental modes in a baratropic star moving on a highly eccentric or parabolic orbit developed elsewhere (see e.g. Press \& Teukolsky 1977, hereafter PT; Lai 1997, IP). This theory is based on consideration of a single pericentre 
passage where an amount of energy $\Delta E$ and an amount of angular momentum $\Delta L$ is transferred from the orbit to the star. These quantities depend on the amplitudes and phases of the pulsation modes before the passage and on the radius of pericentre, $r_{\mathrm{p}}$.

Let us temporarily assume that the amplitude of the pulsation mode before pericentre passage is negligible and the stellar model and the angular velocity of the star are fixed. In this case, it can be shown that the quantities $\Delta E$ and $\Delta L$ are determined by the value of dimensionless parameter (PT)

$\eta=\sqrt{\frac{m}{M}\left(\frac{r_{\mathrm{p}}}{R_{\mathrm{wd}}}\right)^{3}}$,

where $r_{\mathrm{p}}$ is the pericentre distance. These quantities can be naturally considered as being made up of two parts: 1) a quasi-static part determined by irreversible loss of total energy of the system during the pericentre passage and 2) a dynamic part (due to the so-called dynamic tide) determined by excitation of the normal modes of the star.

\subsection{Quasi-static tides}

The quasi-static part of the energy transfer $\Delta E_{q-s}$, can be written in the form: $\Delta E_{q-s} \approx \gamma E_{*} / \eta^{5}$, where $E_{*}=G m^{2} / R_{\mathrm{wd}}$ is some "reference" energy associated with the star and the dimensionless coefficient $\gamma$ is determined by the dissipative processes operating in the star. An explicit expression for $\gamma$ can be found in IP. During the flyby energy loss occurs as a result of viscous dissipation in the bulk of the white dwarf and in its convective envelope and also due to emission of gravitational waves. Since the coefficient of dynamic viscosity in the bulk of the white dwarf appears to be very small $\left(\sim 10^{4}-10^{6}\right.$ in cgs units, e.g. Chugunov \& Yakovlev 2005, and references therein) and convective envelopes of white dwarfs also have a rather small estimated turbulent viscosity and a very small relative mass, their respective contributions to the coefficient $\gamma$ are quite small. The main contribution appears to come from the emission of gravitational waves generated by time dependent perturbation of the white dwarf (see Osaki \& Hansen 1973, hereafter OH). However, a simple estimate shows that the value of $\gamma$ determined by this process leads to a value of $\Delta E_{q-s}$ much smaller than that associated with dynamic tides, for relevant values of $\eta$. Therefore, the overall contribution of quasi-static tides appears to be negligible and will not be considered further.

\subsection{Dynamic tides}

Now let us consider dynamic tides associated with the fundamental quadrupole mode of pulsation. As was shown by IP for the case of a slowly rotating star, the quadrupole mode propagating in the direction of orbital motion (the so-called prograde mode) determines the exchange of energy between the orbit and the star provided that the angular frequency of the star, $\Omega_{\mathrm{r}}$, is smaller that its "equilibrium" value $\Omega_{\mathrm{eq}} \approx(2+\ln \eta) \Omega_{*} / \eta$, where $\Omega_{*}=\sqrt{G m / R_{\mathrm{wd}}^{3}}$ is a "reference" frequency associated with the star. As it will be shown later, in our problem a typical value of $\eta \sim 4$, and the equilibrium angular frequency $\Omega_{\mathrm{eq}} \approx 0.8 \Omega_{*}$ is formally larger than the angular frequency at rotational break-up of the star, $\Omega_{\mathrm{br}} \approx 0.5 \Omega_{*}$. Although the theory leading to this conclusion is not valid at such high rotation rates, it seems reasonable to assume that the energy exchange is mainly determined by the prograde mode unless a rate of rotation very close to $\Omega_{\mathrm{br}}$ is reached. Considering excitation of only this mode and assuming that the star is not perturbed before pericentre passage, it easy to see from results given in IP that the energy gained by the star per unit of mass, $\Delta E_{T}$ can be expressed as

$\Delta E_{T} \approx 0.7 \phi^{-1} \Psi^{-1} \frac{G m}{R_{\mathrm{wd}}}$,

where we use the fact that the eigenfrequency of the quadrupole $f$ mode for a non-rotating $n=1.5$ polytrope $\omega_{f} \approx$ $1.445 \Omega_{*}$ and the value of dimensionless "overlap" integral $Q_{f} \approx$ 0.5 calculated in, e.g., Lee \& Ostriker (1986). The quantity $\phi(\eta)$ characterises the strength of the tidal interaction for a nonrotating star and $\Psi$ determines correction to the energy exchange due to rotation, being unity for a non rotating star (see below). Explicitly, we have

$\phi(\eta)=\eta^{-1} \exp (2.74(\eta-1))$

where $\eta$ is given by Eq. (6). When $\eta \sim 1-2$, the white dwarf is either tidally disrupted or stripped, and the linear theory of dynamic tides cannot be used (see e.g. Frolov et al. 1994; Ivanov \& Novikov 2001, and references therein). As it will be shown later, typically, $\eta \sim 4-5$ for white dwarfs undergoing tidal circularisation. Since the energy gain decreases exponentially with $\eta$ the use of the linear theory seems to be justified in our case.

\subsection{Direct capture or tidal disruption}

The characteristic pericentre distance below which tidal disruption is expected is determined as the distance where the tidal force acting on an element of the star near its surface is comparable to the gravitational force due to the star itself. Thus it is given by

$r_{T}=\left(\frac{M}{m}\right)^{1 / 3} R_{\mathrm{wd}} \approx 1.5 \times 10^{10} \lambda \mu^{-1 / 3} M_{4}^{1 / 3} \mathrm{~cm}$,

where $M_{4}=M /\left(10^{4} M_{\odot}\right)$. Using this we can rewrite Eq. (6) in another form

$\eta=\left(\frac{J}{J_{T}}\right)^{3}$

where $J_{T}=\sqrt{2 G M r_{T}}$ is the specific orbital angular momentum of an orbit with pericentre distance equal to $r_{T}$. From Eq. (10) it follows that when $J \approx$ const. $\eta \propto J^{3}$ is also approximately conserved.

The black hole mainly tidally disrupts the white dwarfs provided that its mass is sufficiently small. To see this we note that the specific orbital angular momentum of stars directly captured by the black hole must be smaller than $J_{\text {cap }}=4 G M / c$. Define the "critical" mass $M_{\text {crit }}$ by the condition that $J_{\text {cap }}=J_{T}$ when $M=M_{\text {crit }}$. One obtains

$M_{\text {crit }}=1.4 \times 10^{4} \lambda^{3 / 2} \mu^{-1 / 2} M_{\odot}$

When $M<M_{\text {crit }}$, the specific angular momentum $J_{T}$ exceeds $J_{\text {cap }}$ so it determines the boundary of the loss cone. Thus $J_{\text {cone }} \approx$ $J_{T}$ and, accordingly, $\eta \approx \Theta^{3}$ (recall that $\Theta$ is defined as $\Theta=$ $\left.J / J_{\text {cone }}\right)$.

\subsection{Effect of stellar rotation}

As indicated above, the rotation rate of the star has an important effect in determining the strength of the tidal interaction. From 
the results given in IP it follows that the corresponding correction factor $\Psi$ entering in Eq. (7) has the form

$\Psi \approx \exp \frac{4 \sqrt{2}}{3} \tilde{\Omega}_{r} \eta$,

where $\tilde{\Omega}_{r}=\Omega_{r} / \Omega_{*}, \Omega_{r}$ is the angular velocity of the star and we recall that $\Omega_{*}=\sqrt{\frac{G m}{R_{\mathrm{wd}}^{3}}}$ characterises a typical frequency of stellar pulsation associated with the fundamental mode. It is important to note that Eq. (12) has been formally derived assuming that $\tilde{\Omega}_{r} \ll 1$. However, we are going to use it later on to make our estimates implying that it is approximately valid when $\Omega_{\mathrm{r}}<\Omega_{\mathrm{br}} \approx 0.5 \Omega_{*}$. Note that this assumption may be justified in part by results of calculations of Managan (1986) who considered normal modes of a fast rotating $n=1.5$ polytrope and of Clement (1989) who considered those of a fast rotating $15 M_{\odot}$ star. In both cases it was found that the eigen frequency of the prograde $f$ mode has an approximate linear dependence on the frequency of rotation until an angular frequency very close to $\Omega_{\mathrm{br}}$ is reached. The linear dependence of the eigen frequency on the frequency of rotation appears to be one of the most important factors leading to Eq. (12).

\subsection{The effect of many pericentre passages and the criterion for stochastic instability}

Let us consider a sequence of many pericentre flybys assuming that the energy per unit mass contained in modes of oscillation, $E_{m}$, does not decay significantly between successive pericentre passages. In this case there is interference between the preexisting wave perturbation in the star and the wave excited in the vicinity of pericentre as a result of tidal perturbation. In this case, simple addition of (7) to $E_{m}$ after the pericentre passage to obtain the new $E_{m}$ needs further justification. In this context it is important that the orbital period $P_{\text {orb }}$ is changing with time as a result of tidal interaction and loss of orbital energy due to emission of gravitational waves ${ }^{2}$, and therefore the the phase change of the oscillating mode generated between two successive pericentre passages, $\Phi=\omega_{f} P_{\text {orb }}$, is also changing with time. When the change of $\Phi$ during one orbital period, $\delta \Phi=\omega_{f} \delta P_{\text {orb }}$ is larger than $2 \pi$, the mode phase at the time of the next pericentre passage is essentially a random quantity, and accordingly, the mode energy per unit mass $E_{\mathrm{m}}$ evolves as a stochastic variable. In this case we can add expression (7) to $E_{\mathrm{m}}$ at each pericentre passage on average, assuming that all resulting expressions are valid in some statistical sense being averaged over many realisations of the stochastic process. The process of stochastic evolution of the mode energy will be refereed to as "stochastic instability".

A condition for the stochastic instability to operate can be readily found from the condition $\omega_{f}\left|\mathrm{~d} P_{\text {orb }} / \mathrm{d} E\right| \Delta E>2 \pi$ and the expression (3) for the orbital period in the form ${ }^{3}$

$a>a_{\mathrm{st}} \approx \frac{(G M)^{3 / 5}}{\left(3 \omega_{f} \Delta E\right)^{2 / 5}}=\lambda \mu^{-3 / 5} M_{4}^{3 / 5}(\Delta \tilde{E})^{-2 / 5} 10^{11} \mathrm{~cm}$,

${ }^{2}$ Note that it is important to distinguish between the effect of emission of gravitational waves by perturbations of the white dwarf and the effect of emission of gravitational waves due to the orbital motion of the star. The former effect is important as a major process of mode energy loss while the latter significantly influences the orbital evolution and may provide a source of gravitational waves during the late stages of orbital evolution, see below.

3 A more accurate expression has been derived in IP. See also this paper for the references on numerical and analytical studies of the stochastic instability. where $a$ is the orbit semi-major axis,

$\Delta \tilde{E} \equiv \Delta \tilde{E}_{T}+\Delta \tilde{E}_{G W} \equiv\left(\frac{R_{\mathrm{wd}}}{G m}\right)\left(\Delta E_{T}+\Delta E_{G W}\right)$

is the dimensionless change of the orbital energy per unit mass, $\Delta E$, in one orbital period, and $\Delta E_{G W}$ is the change of the orbital energy per unit of mass during one orbital period due to emission of gravitational waves, see Eq. (28) below for an explicit expression. As follows from Eq. (14) the condition for the stochastic instability to set in involves two contributions to the change of the orbital energy: $\Delta E_{T}$ associated with tides and $\Delta E_{G W}$ determined by gravitational waves emitted as a result of orbital motion. This differs from the case of tidally interacting stellar binaries where only the tidal contribution is normally considered. In the case of stellar binaries, when the stochastic instability operates, both the orbital binding energy and the energy of the mode of pulsation evolve in a stochastic manner (e.g. Mardling 1995). The fact that $\Delta E_{G W}$ enters into Eq. (14) leads to the possibility of the stochastic instability operating in a situation where orbital evolution is mainly determined by emission of gravitational waves, i.e. $\Delta E_{G W} \gg \Delta E_{T}$, see Sect. 5 and the Appendix. In this limit, our numerical investigations indicate that the orbital binding energy can increase in a way that appears to be fairly regular while the energy of the pulsation mode still tends to increase in an erratic manner. On the other hand, the mode energy ceases to increase when $a<\sim a_{\text {st }}$ and $\Delta E_{T} / \Delta E_{G W}>\sim 0.05$. We have checked that the condition $\Delta E_{T} / \Delta E_{G W}>\sim 0.05$ is fulfilled for parameters typical for our problem.

It is interesting to note that the condition (13) can be also derived from quite a different approach based on the treatment of the tidal interaction as occurring through resonances between the mode frequency $\omega_{f}$ and the changing orbital frequency $\Omega_{\text {orb }}$ of different order $n$, such that $n \Omega_{\text {orb }}=\omega_{f}$. The condition of overlap of these resonances given that $\Omega_{\text {orb }}$ is changing is $\delta n=$ $\omega_{f}\left|d \Omega_{\text {orb }}^{-1} / d E\right| \Delta E>1$. This is equivalent to the condition (13).

\section{Gravitational wave emission/non-linear effects, characteristic time scales and mode amplitude limitation}

As we have mentioned above the most important linear decay channel of the fundamental mode appears to be through emission of gravitational waves. The corresponding decay time of the fundamental mode has been calculated by $\mathrm{OH}$ for two models of iron white dwarfs. The results can be extended to the range of masses and radii appropriate for "standard" CO white dwarfs with help of the expression for the gravitational wave luminosity, $L_{G W}$, produced by the oscillating mode and the resulting decay time can be expressed as

$t_{\mathrm{dec}} \approx 1.5 \times 10^{2} \lambda^{4} \mu^{-3} \mathrm{yr}$.

Note we define the decay time as $t_{\mathrm{dec}}=E_{m} / L_{G W}$ which gives a value of $t_{\mathrm{dec}}$ which is a factor of two smaller compared to that of $\mathrm{OH}$.

In this situation the mode energy can attain a large value as a result of the cumulative effect of multiple tidal interactions occurring at pericentre passage. At high amplitudes non linear effects may lead to additional dissipation and mode decay. We characterise such effects by adopting a mode decay time scale $t_{n l}$ which is a function of the mode energy. The non linear theory for these pulsation modes is not yet adequately developed to provide a form for $t_{n l}$. Therefore, we regard this as a free parameter and consider two limiting cases: a) the time $t_{n l}$ is assumed 
to be large compared to $t_{\mathrm{dec}}$, being taken to be comparable to $t_{\mathrm{ev}}$, the actual time scale of orbital evolution which is the smaller of the times required to change the orbit significantly through tidal interaction or to make it evolve as a result of the emission of gravitational waves induced by that orbital motion, see below Eq. (22). Assuming that all quantities characterising the mode of pulsation and the star evolve on the time scale of order of $t_{\mathrm{ev}}$, we estimate below that the condition $t_{n l} \sim t_{\mathrm{ev}}$ is equivalent to the condition that the rotational angular momentum of the star is the same order of magnitude as the angular momentum corresponding to the mode, see Eq. (18) below. Thus, in this situation the degree of non-linearity adjusts in a way that does not allow the mode angular momentum to significantly exceed that in the stellar rotation. b) The time $t_{\mathrm{nl}}$ is assumed to be short compared to both $t_{\mathrm{dec}}$ and the orbital evolution time scale $t_{\mathrm{ev}}$. However, in both cases we shall assume that $t_{n l}$ is much larger than the orbital period of the tidally interacting star. But note that this is not strictly necessary during the first phase of evolution when the semi-major axis is large (see Sect. 4.1 below)

\subsection{Balance of mode energy and angular momentum input due to tides and losses due to gravitational radiation/non linear effects}

Here we estimate the amount of mode energy that is stored in the star when there is a balance between the build-up of mode energy due to the stochastic instability and decay due to the emission of gravitational waves or non linear effects. Provided this balance can occur with a stellar mode angular momentum content that is small enough to avoid break up of the star, it is possible for it to avoid disruption by having to absorb a large amount of released orbital binding energy. In a stationary state the mode energy per unit of mass can be estimated from the balance equation

$E_{m}\left(\frac{1}{t_{\mathrm{dec}}}+\frac{1}{t_{n l}}\right)=\frac{\Delta E_{T}}{P_{\mathrm{orb}}}$

The mode specific angular momentum, $L_{m}$, is related to $E_{m}$ by the standard equation:

$L_{m}=2 \frac{E_{m}}{\omega_{f}}$,

where we take into account that tides mainly excite the quadrupole mode with the azimuthal mode number $m=2$.

The rate of transfer of angular momentum to the star is related to the rate of dissipation of mode energy (e.g. Goldreich \& Nicholson 1989) and thus determined by $t_{n l}$. The rate of transfer of specific angular momentum is $\dot{L}_{*}=L_{m} / t_{n l}$ and accordingly the total specific angular momentum transferred to the star in a time interval $t_{\mathrm{ev}}$ is estimated to be

$L_{*} \sim\left(\frac{t_{\mathrm{ev}}}{t_{n l}}\right) L_{m}$.

Obviously, $L_{*}$ should not exceed the specific angular momentum of the star in a state of uniform rotation with angular frequency corresponding to rotational break-up.

Estimating the moment of inertia of the star per unit of mass as $I \approx 0.2 R_{\mathrm{wd}}^{2}$ and using the standard relation $L_{*}=I \Omega_{*} \tilde{\Omega}_{r}$, from Eqs. (16)-(18) we obtain the associated dimensionless angular velocity of the star to be given by

$\tilde{\Omega}_{r} \approx 7 \frac{t_{\mathrm{dec}}}{\left(t_{n l}+t_{\mathrm{dec}}\right)}\left(\frac{t_{\mathrm{ev}}}{t_{T}}\right)\left(\frac{R_{*} E}{G m}\right)$, where we introduce the characteristic time for tidal build-up of the mode energy

$t_{T} \equiv \frac{E}{\Delta E_{T}} P_{\mathrm{orb}}$

Note that $t_{T}$ so defined can formally be smaller than $P_{\text {orb. It }}$ is obvious that in that case it is the orbital period which plays the role of the characteristic tidal time scale. Substituting Eq. (7) in (20) we can express the tidal time scale in the form

$t_{T} \approx \lambda \mu^{-1}(\phi \Psi) \mathcal{E}^{-1 / 2} r_{1}^{1 / 2} \mathrm{yr}$,

where $r_{1}=r_{a} /(1 \mathrm{pc})$. Note that since $(\phi \Psi) \gg 1$ typically we have $t_{T} \gg 1 \mathrm{yr}$.

In general the time scale of orbital evolution is given by

$t_{\mathrm{ev}} \approx \min \left(t_{T}, t_{G W}\right)$

where $t_{G W}$ is the characteristic time of orbital evolution due to gravitational wave emission explicitly given below, see Eq. (29). As indicated below, for a white dwarf spun up through tidal interaction, gravitational waves emitted as a consequence of orbital motion are more effective than tides at inducing orbital evolution. In this case $t_{\mathrm{ev}} \sim t_{G W} \ll t_{T}$.

Recalling that the break up angular velocity $\Omega_{\mathrm{br}} \approx 0.5 \Omega_{*}$, and assuming that the criterion $\Omega_{r}<\Omega_{\text {br }}$ provides the condition for the white dwarf to survive the circularisation process, we obtain a limitation on the orbital binding energy per unit mass of the white dwarf, during the phase of stochastic instability, to be given by

$E<E_{\mathrm{dis}}=7 \times 10^{-2} \frac{\left(t_{n l}+t_{\mathrm{dec}}\right)}{t_{\mathrm{dec}}}\left(\frac{t_{T}}{t_{\mathrm{ev}}}\right) \frac{G m}{R_{\mathrm{wd}}}$.

This equation leads to a constraint that the orbital binding energy of the white dwarf should not be too high while mode energy input as implied by the existence of stochastic instability is occurring. The condition (23) can be simplified when $t_{n l}$ is either large or small. For case a) when $t_{n l} \approx t_{\mathrm{ev}}$ the condition (23) can be written as

$\mathcal{E}<\mathcal{E}_{\mathrm{dis}} \equiv\left(\frac{r_{a}}{G M}\right) E_{\mathrm{dis}}=7 \times 10^{-2} \frac{m}{M} \frac{r_{a}}{R_{\mathrm{wd}}} \frac{t_{T}}{t_{\mathrm{dec}}}$,

where we recall that the dimensionless energy $\mathcal{E}$ is related to the orbital binding energy per unit mass $E$ as $\mathcal{E}=\left(r_{a} / G M\right) E$. This condition can be reformulated as setting an upper bound on the allowed mode energy per unit mass as

$E_{m}<7 \times 10^{-2} \frac{G m}{R_{\mathrm{wd}}}$

Note that in case a) the angular momentum transferred to stellar rotation is approximately equal to that associated with the mode of pulsation. Therefore the condition $t_{n l} \approx t_{\mathrm{ev}}$ can be regarded as a condition for equipartition of the angular momentum associated with the pulsation mode and that associated with the stellar rotation.

In the opposite limit corresponding to case b) we have $t_{n l} \ll$ $t_{\mathrm{dec}}, t_{\mathrm{ev}}$. Then Eq. (23) gives

$\mathcal{E}<\mathcal{E}_{\mathrm{dis}}=7 \times 10^{-2} \frac{m}{M} \frac{r_{a}}{R_{\mathrm{wd}}} \frac{t_{T}}{t_{\mathrm{ev}}}$.

In this case the energy put into internal energy of the star, $E_{\text {int }}$, through dissipation is much larger than the mode energy, $E_{m}$, 
and we can express (26) as leading to an upper bound for $E_{\text {int }}$ in a form analogous to that given by (25)

$E_{\mathrm{int}}<7 \times 10^{-2} \frac{G m}{R_{\mathrm{wd}}}$.

We remark that this condition, obtained from considerations of the stellar rotation, is equivalent to specifying a limit on the allowed increase of the internal energy. As almost all of the orbital binding energy is converted to internal energy of the star in case b), the same constraint would occur if the initial evolutionary phase involving stochastic instability were replaced by one in which the energy input into oscillations during each pericentre passage was dissipated before the next one. Thus, as indicated in Sect. 4 above, the condition that $t_{n l}$ should be less than the orbital period is not strictly necessary during the initial evolutionary phase following tidal capture (see also IP).

Note too that we have neglected the change of the white dwarf radius due to tidal heating. Assuming this change is modest, we can take it into account by considering the white dwarf to have a slightly smaller mean density when discussing conditions for safe circularisation, see the next section.

\subsection{Effect of gravitational radiation on the orbit}

The change of orbital energy per unit mass due to emission of gravitational waves generated by orbital motion of the star can be obtained from results given by Peters (1964). In the case of a highly eccentric orbit, the change per orbital period, which is mainly induced at pericentre may be written in the form

$$
\begin{aligned}
\Delta \tilde{E}_{G W} & =3.8 \times 10^{-3}\left(\frac{J}{J_{\text {cap }}}\right)^{-7}\left(\frac{c^{2} R_{\mathrm{wd}}}{G M}\right) \\
& \approx 8 \times 10^{-4} \eta^{-7 / 3} \lambda^{-5 / 2} \mu^{7 / 6} M_{4}^{4 / 3},
\end{aligned}
$$

and the corresponding evolution time can be expressed in terms of $t_{T}$ as

$$
t_{G W}=\left(\frac{\Delta \tilde{E}_{T}}{\Delta \tilde{E}_{G W}}\right) t_{T} .
$$

Since the corresponding quantity induced by tides, $\Delta E_{T}$, decreases with $\eta$ exponentially and $\Delta E_{G W}$ as an inverse power of $\eta$, there is a certain value of $\eta$ where $t_{T}=t_{G W}$. At larger values of $\eta$ the orbital evolution is mainly determined by emission of gravitational waves. For non rotating stars (i.e. setting $\Psi=1$ ) this value of $\eta$ is approximately equal to $\sim 5.3$ for a "high density" white dwarf and $~ 5.6$ for a "low density" white dwarf. As we have mentioned above and will discuss below, a typical value of $\eta$ for the stars undergoing tidal circularisation is of the order of $\sim 4$ and always smaller than $\sim 5$. Taking into account that $t_{T}$ increases very rapidly with this parameter, we can conclude that tidal interactions play the most significant role in the formation of a flow of stars undergoing orbital circularisation.

On the other hand, the star is spun up by tides during ongoing orbital circularisation and $t_{T}$ is increased as a result. Therefore, at a later stage, the orbital evolution may be governed by emission of gravitational waves when the orbital binding energy is sufficiently large, depending on parameters of the white dwarf and of the star cluster. Moreover, when the binding energy exceeds the value corresponding to the onset of stochastic instability, the tidal response becomes quasi periodic and ineffective so that the orbital evolution is solely determined by the emission of gravitational waves.

\section{Conditions for safe circularisation or tidal disruption}

Before going on to estimate of the rate of tidal capture and circularisation we use the results of the previous sections to examine more closely the conditions required for mode amplitude limitation due to gravitational wave emission to enable safe circularisation with the possibility of tidal heating, inflation and disruption avoided.

During the early evolutionary phases, subsequent to tidal capture, the star's orbit is highly eccentric, and the orbital angular momentum is approximately conserved during the orbital evolution while the semi-major axis/binding energy as well as the angular velocity of the star change with time (see e.g. IP). When tides dominate over the emission of gravitational waves induced by orbital motion, the evolution time scale of the semimajor axis is given by Eq. (20) and we have $t_{\mathrm{ev}} \approx t_{T}$. When gravitational radiation controls the orbital evolution, which happens at large enough orbital binding energy, we have $t_{\mathrm{ev}} \approx t_{G W}$ where $t_{G W}$ is given by Eq. (29). For a given value of the orbital angular momentum, the orbital evolution time $t_{\mathrm{ev}}$ depends on the orbital energy $E$, and thus, on the star's semi-major axis $a$. This dependence can be found from Eqs. (19)-(25) and (29). For illustrative purposes we plot this dependence as well as that of other characteristic time-scales in Figs. 1 and 2 for a "low density" white dwarf orbiting around a $10^{4} M_{\odot}$ black hole. The cusp size is taken to be $1 \mathrm{pc}$. All dependencies are calculated in assumption that the stochastic instability operates for the shown range of binding energies.

In Fig. 1 the characteristic time-scales corresponding to a star with $\eta=5$ are shown. The horizontal line indicates the decay time scale of the stellar pulsations due to the emission of gravitational waves, $t_{\mathrm{dec}}$, given by Eq. (15). The inclined dotted line indicates the dependence of the characteristic time $t_{G W}$ on $\mathcal{E}$ and the inclined dashed and dot-dashed lines show the dependence of $t_{T}$ on $\mathcal{E}$ for $\Omega_{r}=\Omega_{\mathrm{br}}=0.5 \Omega_{*}$ (dashed line) and $\Omega_{r}=0$ (dot-dashed line). We recall that the timescale $t_{T}$ applies to the evolutionary phase just after tidal capture which can exhibit stochastic instability. It may not be applicable to the later stages, when $\mathcal{E}$ is large, and the conditions for additive impulsive energy inputs at pericentre passage are not satisfied, see discussion below.

For a given value of $\mathcal{E}$, the dot-dashed line corresponding to a non rotating star, gives the smallest possible value of $t_{T}=$ $t_{\min }$. For fixed $\mathcal{E}$, stars with values $t_{T}$ exceeding that given by the dashed line, would have angular velocities exceeding $\Omega_{\mathrm{br}}$, and so would be disrupted. Therefore the dashed curve gives the largest allowed value of $t_{T}=t_{\max }$.

The solid curves indicate the evolution of $t_{\mathrm{ev}}$ with $\Omega_{r}$ which is given by Eq. (19). The two different curves correspond to the different assumptions about the value of the mode decay time due to non-linear effects, $t_{n l}$. The curve which is uppermost at small values of $\mathcal{E}$ corresponds to the case a) where we assume that $t_{n l} \approx t_{\mathrm{ev}}$ while the other solid curve corresponds to the case b) for which $t_{n l}$ is much less than both $t_{\mathrm{dec}}$ and $t_{\mathrm{ev}}$. One can see from Figs. 2 and 3 that the behaviour of the characteristic time-scales is rather similar for these two cases. When $\mathcal{E}$ is very small, rotation of the star is small, and both curves are close to the dot-dashed line. Also, for small values of $\mathcal{E}$ we have $t_{T}=t_{\mathrm{ev}}$.

When $\mathcal{E}$ increases, the star is spun up by tides and $t_{T}$ gets larger. When $\mathcal{E}$ attains a value $\mathcal{E}_{G W} \sim 10^{4}$ the solid curves, which for smaller values, correspond to tidally driven evolution cross the dotted line representing evolution controlled by the gravitational radiation time scale $t_{G W}$. For larger values of $\mathcal{E}$, the 


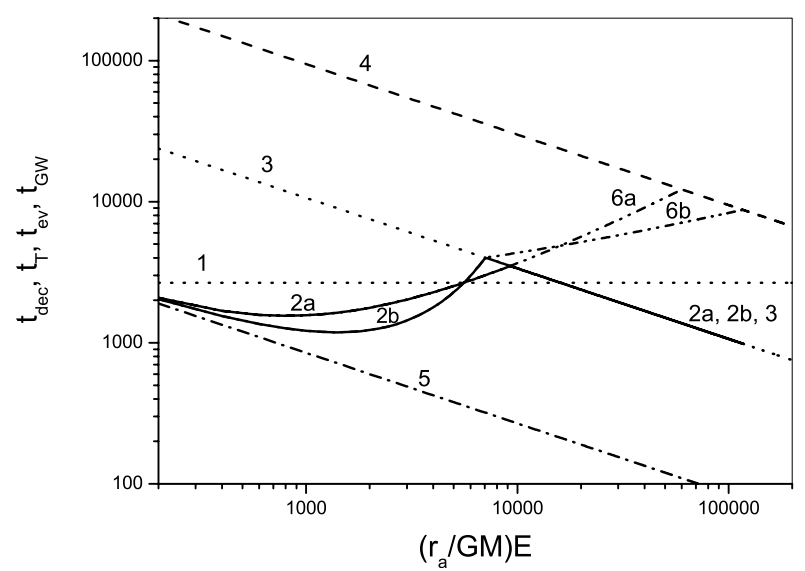

Fig. 2. Characteristic time scales in years as functions of the dimensionless orbital energy $\mathcal{E}$. Plotted here are $t_{\mathrm{dec}}$, the decay time of the pulsation mode due to emission of gravitational waves associated with the stellar pulsation, $t_{\mathrm{ev}}$, the time scale for evolution of the orbit, $t_{T}$, the time scale of build-up of the mode energy and $t_{\mathrm{GW}}$, the time scale of orbital evolution due to emission of gravitational waves induced by orbital motion. The horizontal dotted line 1 represents $t_{\mathrm{dec}}$. The two solid curves $2 \mathrm{a}$ and $2 \mathrm{~b}$ represent $t_{\mathrm{ev}}$ calculated assuming that the stellar rotation frequency is given by Eq. (19). The curve 2a taking on larger values at small values of $\mathcal{E}$ represents the case $t_{n l} \approx t_{\mathrm{ev}}$, and the other curve represents the case $t_{n l} \ll t_{\mathrm{ev}}$. The inclined dotted line 3 represents $t_{G W}$ which becomes equal to $t_{\mathrm{ev}}$ at the larger binding energies where gravitational radiation controls the orbital evolution. The dashed and dot dashed lines 4 and 5 represent $t_{T}$ calculated for $\Omega_{r}=\Omega_{\mathrm{br}}$ and $\Omega_{r}=0$, respectively. The dot dot dashed curves $6 \mathrm{a}$ and $6 \mathrm{~b}$ represent $t_{T}$ calculated for the star rotating with angular frequency given by Eq. (19) for values of binding energies larger than that corresponding to the intersection of $t_{\mathrm{ev}}$ and $t_{\mathrm{GW}}$. For smaller values of energies we have $t_{T}=t_{\mathrm{ev}}$. Note the slower evolution due to tides for the faster rotation rate. This results in gravitational radiation ultimately controlling the orbital evolution. All curves are calculated for $\mu=0.6, \lambda=1.4, \eta=5, r_{1}=1$ and $M_{4}=1$.

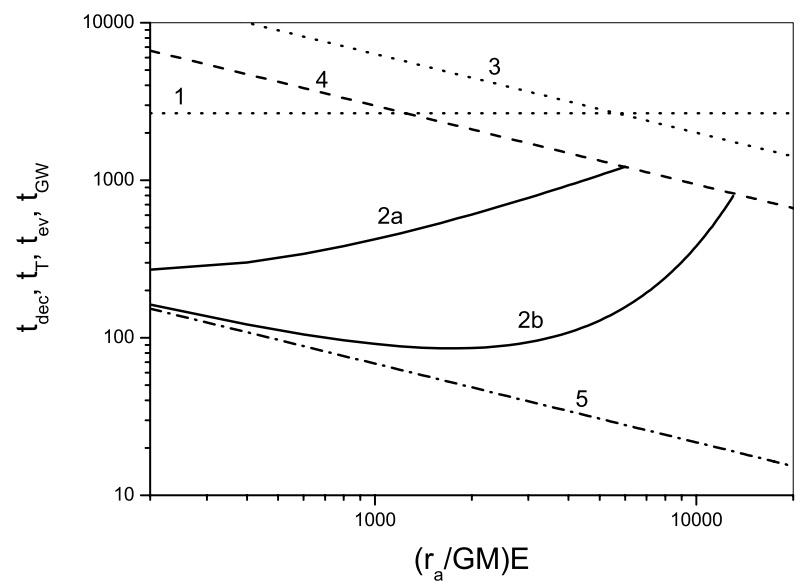

Fig. 3. Same as Fig. 1 but for $\eta=4$. Note that in this case we have $t_{\mathrm{ev}}=t_{T}$ for the whole range of binding energies shown.

orbital evolution is mainly determined by emission of gravitational waves, and we have $t_{\mathrm{ev}}=t_{G W}<t_{T}$. In this regime the star continues to spin up by tides, and the tidal time scales shown by dot dot dashed curves get larger with increasing of $\mathcal{E}$. When the dot dot dashed curves cross the inclined dashed line, the binding energies are equal to $E_{\text {dis }}$ given by Eqs. (24) and (26), and, according to our criterion, the star is disrupted at energies $E \sim E_{\mathrm{dis}}$.

In Fig. 2 we show the same quantities calculated for a star with a smaller value of orbital angular momentum corresponding to $\eta=4$. In this case the evolution is always controlled by tidal effects and $t_{\mathrm{ev}}=t_{T}$ for the whole range of energies shown $\mathcal{E} \leq$ $\mathcal{E}_{\text {dis }}$ and, accordingly, the the dot dot dashed curves coincide with the solid curves.

As follows from this discussion and Figs. 2 and 3, the white dwarf has the possibility of surviving the tidal evolution when the energy scale corresponding to the onset of the stochastic instability $E_{\mathrm{st}}=G M /\left(2 a_{\mathrm{st}}\right)$ (see Eq. (13)) is smaller than $E_{\text {dis }}-$ the energy corresponding to break-up rotation. When $E_{\mathrm{st}}<E_{\mathrm{dis}}$, disruption of the star is avoided during the phase of stochastic instability. In this case, when $E>E_{\mathrm{st}}$ the orbital evolution proceeds mainly through emission of gravitational waves. Therefore, for our purposes it is very important to establish under what conditions the inequality $E_{\mathrm{st}}<E_{\text {dis }}$ holds.

As can be seen from Figs. 2 and 3, there are four different possibilities: case a1) where $t_{n l} \approx t_{\mathrm{ev}}$ and tides determine the orbital evolution when the orbital energy of the star is close to $E_{\mathrm{dis}}$; case a2) where $t_{n l} \approx t_{\mathrm{ev}}$ and the gravitational radiation determines the orbital evolution; case b1) when $t_{n l} \ll t_{\mathrm{ev}}$ and tides determine the evolution, and the case b2) where $t_{n l} \ll t_{\mathrm{ev}}$ and gravitational radiation controls the evolution. Note that it is straightforward to see that case b1) is always associated with disruption of the star. Indeed, in this case the factor $t_{T} / t_{\mathrm{ev}}$ entering Eq. (26) is of order of unity. That means that the energy $E_{\text {dis }}$ is always small when compared to $E_{\mathrm{st}}$, for typical parameters of the system. Therefore, the condition $E_{\mathrm{st}}<E_{\mathrm{dis}}$ cannot be satisfied in this case and the case b1) is not considered further. On the other hand, in the opposite case b2) the factor $t_{T} / t_{\mathrm{ev}}$ can be large when estimated at energies of the order of $E_{\mathrm{dis}}$, see Fig. 1, and the condition $E_{\mathrm{st}}<E_{\text {dis }}$ can be fulfilled, see Appendix for details. In Appendix it is also shown that circularisation is possible for the cases a1) and a2) provided that the orbital angular momentum of the star and, accordingly, $\eta$, is sufficiently large. Thus, circularisation can, in principal, be achieved for the cases a1), a2) and b2).

The condition $E_{\mathrm{st}}<E_{\mathrm{dis}}$ can also be reformulated in terms of semi-major axes. Using the expression (23) we can easily find the characteristic semi-major axis $a_{\text {dis }}$ corresponding to the binding energy per unit mass $E_{\text {dis }}$

$a_{\mathrm{dis}}=\frac{r_{a}}{2 \mathcal{E}_{\mathrm{dis}}}=5 \times 10^{13}\left(\frac{t_{\mathrm{ev}}}{t_{T}}\right)\left(\frac{t_{\mathrm{dec}}}{t_{n l}+t_{\mathrm{dec}}}\right) \lambda \mu^{-1} M_{4} \mathrm{~cm}$.

As we discussed above when $a<a_{\text {dis }}$ and the condition for the stochastic instability (13) holds, emission of gravitational waves/non linear effects cannot significantly reduce the energy and angular momentum of the mode and the mode amplitude build-up persists. In such a situation a white dwarf is likely to undergo intensive tidal heating which may possibly lead to disruption of the star. Therefore, the condition derived from (30) together with corresponding conditions for onset of the stochastic instability will be used in order to discriminate between orbital evolutionary tracks leading to disruption of the star and the tracks eventually leading to formation of close quasi-circular orbits around the black hole.

When the non linear dissipation time scale $t_{n l}$ is small (the cases b1 and b2), $a_{\mathrm{dis}}$ does not depend on $t_{n l}$ and we have

$a_{\mathrm{dis}}=\frac{r_{a}}{2 \mathcal{E}_{\mathrm{dis}}}=5 \times 10^{13}\left(\frac{t_{\mathrm{ev}}}{t_{T}}\right) \lambda \mu^{-1} M_{4} \mathrm{~cm}$.

When $t_{n l} \approx t_{\mathrm{ev}}$ (the cases a1 and a2) we can obtain an explicit expression for $a_{\text {dis }}$ with the help of (21):

$a_{\mathrm{dis}}=\frac{r_{a}}{2 \mathcal{E}_{\mathrm{dis}}}=4.4 \times 10^{16} \lambda^{8 / 3} \mu^{-2} M_{4}^{1 / 3}(\phi \Psi)^{-2 / 3} \mathrm{~cm}$. 
The condition $a_{\mathrm{st}}>a_{\mathrm{dis}}$ ( or $E_{\mathrm{st}}<E_{\mathrm{dis}}$ ) can be written as a condition for the parameter $\eta$ to be larger than a certain value. For our discussion below it is more convenient to use the quantity $\phi(\eta)$ defined in Eq. (8) instead of $\eta$. Depending on which case is considered (i.e. a1, a 2 or b2), the inequality $a_{\mathrm{st}}>a_{\text {dis }}$ can be written as a condition that the quantity $\phi(\eta)$ is larger than its values $\phi_{1}, \phi_{2}$ and $\phi_{3}$ corresponding to the equality $a_{\mathrm{st}}=a_{\mathrm{dis}}$ for the respective cases. These values are explicitly obtained in the Appendix, see Eq. (A.5) for the case a1, Eq. (A.7) for the case a2 and Eq. (A.10) for the case b2.

\section{The rate of tidal capture by the black hole}

In order to estimate the rate of tidal capture by the black hole we assume that the stars in the cusp are slowly rotating. Therefore, at the beginning of orbital circularisation the effects of rotation are not important so we neglect it when estimating the rate of capture into circularising orbits, setting $\Psi=1$ in the expression (21) for the time scale of orbital evolution. Then from the discussion in Sect. 5 and data plotted in Figs. 2 and 3 it follows that during this phase orbital energy loss resulting from tides is more important than that due to gravitational radiation.

The rate of capture into circularising orbits, as a function of initial binding energy per unit mass of the star, depends on whether the tidal time scale (21) is larger than the orbital period $P_{\text {orb }}(\mathcal{E})$. Equating (21) to (3) we find the binding energy per unit mass $\mathcal{E}_{1}$ where these two times are equal to be given by

$\mathcal{E}_{1}=0.7 \phi^{-1}(\eta)\left(\frac{r_{a}}{R_{\mathrm{wd}}} \frac{m}{M}\right)$,

and we recall the relation between $\eta$ and the $\Theta=J / J_{\text {cone }}$ that applies when the condition $M<M_{\text {crit }}$ as given by (11) is fulfilled is $\Theta \approx \eta^{1 / 3}$.

\subsection{Balance of time scales for tidal evolution and orbital evolution due to distant encounters}

At first we assume that $\mathcal{E}>\mathcal{E}_{1}$ for a range of angular momenta of interest. A star gets tidally captured by the black hole when the characteristic time scale of evolution of its angular momentum due to distant gravitational encounters with other stars in the cluster

$t_{J} \sim \frac{J^{2}}{\left(\Delta j_{2}\right)^{2}} P_{\mathrm{orb}}$,

is larger than the tidal time scale $t_{T}$ (recall that the dispersion of angular momentum $j_{2}^{2}$ is given by Eq. (5)). Equating $t_{J}$ and $t_{T}$ we find the size of the "tidal circularisation" loss cone in angular momentum space as a function of the dimensionless binding energy $\mathcal{E}$,

$J_{\mathrm{T} . \mathrm{C}}=\sqrt{\left(\Delta j_{2}\right)^{2} \frac{t_{T}}{P_{\mathrm{orb}}}}$.

Note that Eq. (35) defines $J_{\text {T.C. }}$ implicitly since this quantity enters the right hand side through the dependence of $t_{T}$ on $\eta$. To deal with this we define $\Theta_{\mathrm{T} . \mathrm{C}}=J_{\mathrm{T} . \mathrm{C}} / J_{T}$, where we recall that $J_{T}=\sqrt{2 G M r_{T}}$. Then we have $\Theta_{\mathrm{T} . \mathrm{C}}=\eta^{1 / 3}$, see Eqs. (9) and (10). Using the above, an equation for $\Theta_{\text {T.C. may be obtained }}$ from Eqs. (5), (20) and (35) in the form

$\Theta_{\text {T.C. }}=1.14 \mu^{-1 / 3} M_{4}^{5 / 6} \Lambda_{*}^{1 / 2} \phi^{1 / 2}\left(\Theta_{\text {T.C. }}^{3}\right) \mathcal{E}^{-3 / 4}$, where $\Lambda_{*}=\Lambda_{2} / 8.5$. It follows from Eq. (20) that in order to find $\Theta_{\text {T.C. }}$ and hence the size of the tidal loss cone we need to evaluate the ratio $\Delta E_{T} / E$ when $J=J_{\text {T.C. }}$ This quantity grows from small values as the orbital period increases from small values, and accordingly the binding energy decreases, until it reaches unity when $t_{J}=P_{\text {orb }}$. At that point Eq. (33) applies as well as the condition that

$J_{\text {T.C. }}^{2}=\left(\Delta j_{2}\right)^{2}$.

These two equations define a critical energy $E_{\text {crit }}$.

When $E<E_{\text {crit }}$ the tidal evolution time scale is approximately equal to the orbital period and therefore the size of the tidal circularisation loss cone is determined by Eq. (33) which is, in this case, considered as an implicit equation for $\Theta \equiv J_{\text {T.C. }} / J_{\text {cone }}$ for a given value of $\tilde{E}$. Stars with these energies which have $J<J_{\text {T.C. }}$ calculated in this way have relatively large energy changes induced within a single orbit.

When $E>E_{\text {crit }}$ the number of stars tidally captured by the black hole per unit of time, with typical energies of order of some $E, \dot{N}_{\text {T.C. }}(E)$, can be estimated to be

$\dot{N}_{\text {T.C. }}(E) \sim N_{\text {wd }}^{0}\left(E, J_{\text {T.C }}\right)\left(E J_{\text {T.C }} /\left(\Lambda_{1} t_{T}\right)\right.$,

where we assume that the distribution function of the white dwarfs is given by Eq. (4) ${ }^{4}$. Using Eqs. (2), (5) and (35) we see that, in this case,

$\dot{N}_{\text {T.C. }} \propto\left(\Delta j_{2}\right)^{2} E \propto E^{-3 / 2}$

is mainly determined by stars with small energies. Note that we take into account the fact that $\Lambda_{1}$ depends only logarithmically on the binding energy when deriving the relation (39), see Eq. (35).

For the case $E<E_{\text {crit }}$ it can be easily seen that $t_{J}<t_{T} \approx P_{\text {orb }}$ for any value of $J$. This situation is analogous to the full loss cone regime in the theory of tidally disrupted stars (e.g. Frank \& Rees 1976; Lightman \& Shapiro 1977). In this case the rate of tidally captured stars can be estimated as

$\dot{N}_{\text {T.C. }}(E) \sim N_{\mathrm{wd}}^{0}\left(E, J_{\mathrm{T} . \mathrm{C}}\right) E J_{\mathrm{T} . \mathrm{C}} /\left(\Lambda_{1} P_{\mathrm{orb}}\right) \propto E$,

where here we take into account the fact that the size of loss cone, now implicitly determined by Eq. (33), depends logarithmically and hence slowly on $E$.

\subsection{Determination of the critical binding energy per unit mass}

Thus, the rate of tidal circularisation is mainly determined by energies $E \sim E_{\text {crit }}$ (Novikov et al. 1992), and therefore it important to obtain an explicit expression for the value of $E_{\text {crit. }}$. As we discussed above this value is determined by the condition that the timescale for orbital evolution due to tides be equal to the orbital period while being on the boundary of the tidal loss cone. It is determined by Eqs. (33) and (37) which can be rewritten in the form

$\mathcal{E}_{\text {crit }} \equiv \frac{r_{a}}{G M} E_{\text {crit }}=3 \times 10^{5} \phi_{\text {crit }}^{-1} x$,

${ }^{4}$ Note an alternative form of Eq. (38) has already been provided in other studies, see e.g. Hopman \& Alexander (2005), their Eq. (17) and references therein. To obtain this we write $\dot{N}_{\text {T.C. }}(E) \sim N_{\text {wd }}^{0}\left(E, J_{\text {T.C }}\right) E J_{\text {T.C }} /\left(t_{T} \Lambda_{1}\right)=N_{\text {tot }} /\left(t_{r} \Lambda_{1}\right)$, where $N_{\text {tot }} \equiv$ $N_{\mathrm{wd}}^{0}\left(E, J_{\text {circ }}\right) J_{\text {circ }} E$ characterises the total number of stars having binding energies per unit mass of order of $E$, we introduce the global relaxation time scale $t_{r} \equiv J_{\text {circ }}^{2} P_{\text {orb }} /\left(\Delta j_{2}\right)^{2}$ and use Eqs. (2), (4) and (35). 
where $x=\mu r_{1} /\left(\lambda M_{4}\right)$ and $\phi_{\text {crit }} \equiv \phi\left(\Theta_{\text {T.C. }}^{3}\right)$ is found from Eq. (8) after setting $\eta=\Theta_{\text {T.C. }}^{3}$, together with

$\Theta_{\text {T.C. }}^{2}=\frac{29 \pi}{40} \Lambda_{2} q\left(\frac{r_{a}}{r_{T}}\right) \mathcal{E}_{\text {crit }}^{-5 / 2}$.

Substituting $\mathcal{E}_{\text {crit }}$ from (41) to (42) we obtain a single equation for $\eta$ and thence $\mathcal{E}_{\text {crit }}$ in the form

$\phi_{\text {crit }}=1.7 \times 10^{3} y^{3 / 5} \Theta_{\text {T.C }}^{4 / 5}$

where

$y=\Lambda_{*}^{-2 / 3} \mu^{13 / 9} M_{4}^{-7 / 9} \lambda^{-1} r_{1}$,

and $\Lambda_{*}=\Lambda_{2} / 8.5$. Using the explicit expression for $\phi(\eta)$ and the relation $\eta=\Theta_{\text {T.C. }}^{3}$ we rewrite (43) in another form

$\eta \approx 3.72+0.462 \log \eta+0.219 \log y$.

An approximate solution to Eq. (45) valid for the range of interest of the parameter $y \sim 0.05-10$ with accuracy of order of 10 per cent is

$\eta \approx 4.3+0.22 \log y$,

and

$\phi_{\text {crit }}(\eta) \approx 2 \times 10^{3} y^{3 / 5}$.

As seen from Eq. (47) the size of the tidal circularisation loss cone given by $\Theta_{\mathrm{T} . \mathrm{C}}=\eta^{1 / 3}$ corresponding to the critical energy $E_{\text {crit }}$ is larger than the size of the tidal disruption loss cone given by $\Theta=1$.

This is different from the situation where only the effect of emission of gravitational waves is taken into account as a mechanism for changing the orbital energy. In that case the energy scale analogous $E_{\text {crit }}$ may also be obtained from the requirement that the orbital evolution time $t_{\mathrm{ev}}=t_{G W}$ be equal to the orbital period on the boundary of the corresponding circularisation loss cone. Obviously, our estimate of the circularisation rate is valid only when the size of the circularisation loss cone is larger than the size of "true" loss cone, $J_{\text {cone }}$ corresponding to direct capture or disruption of stars by the black hole. However, for typical parameters expected for gravitational waves to be the dominant process of orbital evolution $\left(M \sim 10^{6} M_{\odot}\right.$ and $\left.r_{1} \sim 1\right)$ the energy scale $E_{\text {crit }}$ is smaller than another energy scale $E_{\text {cone }}$ defined by the condition that the sizes of the circularisation and true loss cones coincide at $E=E_{\text {cone. }}$. When $E>E_{\text {cone }}$ the stars are mainly destroyed by the black hole and the process of circularisation is strongly suppressed. In such a situation the capture rate is mainly determined by energies $E \sim E_{\text {cone }}$ (Hopman \& Alexander 2005). Because of this difference, the tidal circularisation rate in our case, (see Eq. (51) below) differs from the estimate of the capture rate due to emission of gravitational waves provided by Hopman \& Alexander (2005), see also Novikov et al. (1992).

Equation (41) gives

$\mathcal{E}_{\text {crit }} \approx 150\left(\frac{x}{y^{3 / 5}}\right)$.

We have explicitly

$\mathcal{E}_{\text {crit }} \approx 150 \Lambda_{*}^{2 / 5}\left(\frac{r_{1}}{\lambda}\right)^{2 / 5} \mu^{2 / 15} M_{4}^{-8 / 15}$.

It is important to note that the critical energy $\mathcal{E}_{\text {crit }} \gg 1$ for our problem and therefore, the circularisation rate is mainly determined by scales well within the cusp around the black hole. Also, Eq. (46) tells that $\eta \sim 4$ as we have mentioned above.

\subsection{Rate of capture into circularising orbits}

The maximal rate of capture, $\dot{N}_{\text {T.C }}^{\text {max }}$ can be estimated a little more accurately as

$\dot{N}_{\mathrm{T} . \mathrm{C}}^{\max } \sim E_{\text {crit }} \int_{J_{\text {cone }}}^{J_{\text {T.C. }}} \mathrm{d} J \frac{N_{\mathrm{w} . \mathrm{d}}}{P_{\text {orb }}}$,

where $N_{\text {w.d }}$ is given by Eq. (4) with $E=E_{\text {crit }}$ and $J_{\text {cone }}$ is defined in Sect. 2.4. Let us recall that in our problem $J_{\text {cone }}=J_{T}$. Integrating (50) we get

$\dot{N}_{\text {T.C. }}^{\max }=\frac{F}{\sqrt{2} \Lambda_{1}} \delta q^{-1}\left(\frac{r_{T}}{r_{a}}\right) \mathcal{E}_{\text {crit }} \Omega_{a}$,

where

$F=\left(\Theta_{\text {T.C. }}^{2}\left(\ln \Theta_{\text {T.C. }}^{2}-1\right)+1\right) / 4 \approx 0.2$,

and we assume that $\Theta_{\mathrm{T} . \mathrm{C}}=\eta^{1 / 3} \approx 4^{1 / 3}$ in the last equality. In astrophysical units the expression (51) has the form

$\dot{N}_{\text {T.C. }}^{\max } \sim 2.5 \times 10^{-8} \delta_{0.1} \Lambda_{*}^{2 / 3} \Lambda_{8}^{-1} \mu^{-1 / 5} M_{4}^{13 / 10} \lambda^{3 / 5} r_{0.1}^{-21 / 10} \mathrm{yr}^{-1}$,

where $\delta_{0.1}=\delta / 0.1$ and $\Lambda_{8}=\Lambda_{1} / 8$. Note that we use $r_{0.1}=$ $r_{a} /(0.1 \mathrm{pc})=10 r_{1}$ in the last equation assuming that a typical size of the radius of influence in globular clusters is of the order of $0.1 \mathrm{pc}$.

\subsection{Rate of capture of stars that will circularise without disruption}

As discussed in Sect. 5, a white dwarf has a possibility of surviving the process of circularisation, and accordingly, settling on a quasi-circular orbit only when a typical value of the parameter $\eta$ (or equivalently the pericentre distance) is sufficiently large. The tidally captured white dwarfs with formation rate $\dot{N}_{\text {T.C. }}^{\max }$ have a typical value of $\eta$ given by Eq. (46) and, accordingly, $\phi_{\text {crit }}(\eta)$ given by Eq. (47).

According to the discussion in Sect. 5 and the Appendix, such white dwarfs have a considerable survival probability, for cases a1) and a2) corresponding to large $t_{n l}$, when

$\phi_{\text {crit }}>\phi_{\max } \equiv \max \left(\phi_{1}, \phi_{2}\right)$

where $\phi_{1}$ and $\phi_{2}$ are determined by Eqs. (A.5) and (A.7), respectively. For case b2) corresponding to small $t_{n l}$, for survival we require that

$\phi_{\text {crit }}>\phi_{\max }=\phi_{3}$

instead of (54), where $\phi_{3}$ is given by Eq. (A.10).

When the above conditions are not satisfied, the typical binding energy of white dwarfs having a considerable probability of survival, $E_{\mathrm{s}}$, has to be larger than the critical value obtained from Eq. (49) and so their capture rate is reduced by a factor $\left(E_{\mathrm{s}} / E_{\text {crit }}\right)^{3 / 2}$ when compared to that given by Eq. (53) (see Eq. (39)). Accordingly their capture rate is given by

$\dot{N}_{\text {T.C. }}^{s} \approx \dot{N}_{\text {T.C. }}^{\max }\left(E_{\mathrm{S}} / E_{\text {crit }}\right)^{-3 / 2}$.

The binding energy per unit mass, $E_{\mathrm{s}}$, is related to the quantity $\phi_{\max }$ through the "loss cone condition" (36). Taking into account

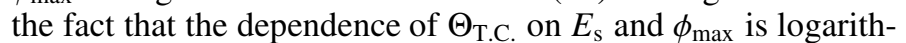
mic, we approximately have $E_{\mathrm{s}}^{3 / 2} \propto \phi_{\max }$, and therefore Eq. (56) can be written as

$\dot{N}_{\text {T.C. }}^{s} \approx \dot{N}_{\text {T.C. }}^{\max }\left(\phi_{\text {crit }} / \phi_{\text {max }}\right)$, 
and accordingly, the rate of formation is suppressed by the factor $\phi_{\text {crit }} / \phi_{\max }$ which is less than unity when conditions (54) and (55) are not satisfied. As indicated below that is likely to be the situation for cases of interest.

We go on to obtain explicit expressions for the rate of capture of white dwarfs that may survive the circularisation process in that case. This rate will be referred to as "the circularisation rate".

First let us consider the case a) for which $t_{n l} \approx t_{\mathrm{ev}}$. As follows from Eq. (A.8) $\phi_{2}>\phi_{1}$ when $M>2 \times 10^{3} M_{\odot}$. Since the systems containing sufficiently massive black holes are likely to provide the bulk of the sources of gravitational radiation, we assume hereafter that $M>2 \times 10^{3} M_{\odot}$ and set, accordingly, $\phi_{\max }=\phi_{2}$. In this case the condition (54) leads to

$r_{0.1}>50 \lambda^{2.1} \mu^{-3} M_{4}^{1.2}$,

where we use Eqs. (A.7) and (44). In the systems of interest this condition is not satisfied, and indeed the circularisation rate is suppressed.

We use Eqs. (44), (47), (53) and (57) to obtain the the suppressed capture or circularisation rate in the form

$\dot{N}_{\text {T.C. }}^{s} \approx 2.3 \times 10^{-9} \delta_{0.1} \mu^{1.6} M_{4}^{0.57} \lambda^{-0.65} r_{0.1}^{-1.5} \mathrm{yr}^{-1}$,

where hereafter we set the logarithmic factors $\Lambda_{*}=\Lambda_{8}=1$. Substituting values of $\lambda=1.4$ and $\mu=0.6$ appropriate for a "low density" white dwarf in (59), we obtain

$\dot{N}_{\text {T.C. }}^{\text {s }} \approx 8.2 \times 10^{-10} \delta_{0.1} M_{4}^{0.57} r_{0.1}^{-1.5} \mathrm{yr}^{-1}$,

and in the case of the "high density" white dwarf with $\lambda=0.84$ and $\mu=1$, we obtain

$\dot{N}_{\text {T.C. }}^{\text {s }} \approx 2.6 \times 10^{-9} \delta_{0.1} M_{4}^{0.57} r_{0.1}^{-1.5} \mathrm{yr}^{-1}$.

Now let us consider case b2) for which $t_{n l} \ll t_{\mathrm{ev}}$, $t_{\mathrm{dec}}$. In this case the condition (55) can be expressed as

$r_{0.1}>10 \lambda^{3} \mu^{-135 / 45} M_{4}^{1 / 9}$,

where we have made use of Eqs. (A.10) and (47). According to Eq. (62) the circularisation rate is equal to the rate of tidal capture (53) when the cusp size is sufficiently large. For large cusp sizes the circularisation rate becomes small due to a strong decrease of the capture rate as it increases (see Eq. (53)). Thus such systems are not likely to contribute significantly to the total number of gravitational wave sources. Therefore, we assume hereafter that the inequality (62) does not apply and multiply (53) by the suppression factor $\left(\phi_{\text {crit }} / \phi_{3}\right)$ in order to obtain the circularisation rate. Thus we have

$\dot{N}_{\text {T.C. }}^{\text {s }} \approx 6.2 \times 10^{-9} \delta_{0.1} \mu^{122 / 75} M_{4}^{37 / 30} \lambda^{-6 / 5} r_{0.1}^{-1.5} \mathrm{yr}^{-1}$.

Substituting values appropriate to the two types of white dwarf in (63), we obtain for the "low density" case

$\dot{N}_{\text {T.C. }}^{\mathrm{s}} \approx 1.8 \times 10^{-9} \delta_{0.1} M_{4}^{1.2} r_{0.1}^{-1.5} \mathrm{yr}^{-1}$,

and

$N_{\text {T.C. }}^{\mathrm{s}} \approx 7.7 \times 10^{-9} \delta_{0.1} M_{4}^{1.2} r_{0.1}^{-1.5} \mathrm{yr}^{-1}$

for the "high density case".

A comparison of Eq. (53) with Eqs. (59)-(61), (63)-(65) shows that our criterion of survival of the white dwarfs during the process of circularisation typically results in an order of magnitude decrease of the circularisation rate. However note that for case b2) when $t_{n l}$ is small, the suppression is not significant for the "high density" white dwarfs..

\section{Probability of detection of a source of gravitational radiation}

The probability of formation of observable sources of gravitational waves is determined by several important factors such as the rate of formation of white dwarfs on circularising orbits calculated above, the subsequent orbital evolution of the white dwarfs, the abundance of globular clusters having sufficiently massive black holes and the properties of a gravitational wave antenna receiving the signal. In what follows we make a rough estimate of the probability assuming that the gravitational wave antenna has characteristics close to what has been indicated for the future LISA space borne gravitational wave antenna.

LISA has its maximal sensitivity in the frequency range $\sim 10^{-2} \mathrm{~Hz}$ for which the dimensionless amplitude of the gravitational waves, $h$, can be as small as

$h \approx 10^{-24} \sqrt{\frac{1 \mathrm{yr}}{T_{\mathrm{obs}}}}$

where $T_{\text {obs }}$ is the time of observation, see e.g. Grishchuk et al. (2001, and references therein). Therefore, for simplicity, we assume that the white dwarf has an orbital period $P_{\text {orb }} \approx 100 \mathrm{~s}$ when emitting the gravitational waves to be received by the antenna. The time $T_{\text {obs }}$ can be smaller than or of the same order as the orbital evolution time $T_{\mathrm{ev}}$ calculated for an orbital period $\sim 100 \mathrm{~s}$, and we assume hereafter that these are equal to each other, $T_{\mathrm{obs}}=T_{\mathrm{ev}}$. In order to estimate $T_{\mathrm{ev}}$ we need to specify a typical value of eccentricity of the orbit, $e$, which, in turn, is determined by whether the orbital evolution is governed by tides or by the emission of the gravitational waves. For such small orbital periods the evolution is likely to be determined by the latter effect and we can use the results obtained by Peters \& Mathews (1963) and Peters (1964) when estimating $e$. To do that we proceed as follows. First we estimate the orbital semi-major axis for an orbit with $P_{\text {orb }}=100$ s from Kepler's law as

$a \approx 4.7 \mu^{1 / 3} \lambda^{-1} r_{T}$

Next we use the dependence of the orbital angular momentum on $e$ obtained by Peters (1964)

$J=0.94 H(e) e^{6 / 19} \eta_{t}^{1 / 3} J_{T}$,

where $H(e)=\left(1+\frac{121}{304} e^{2}\right)^{435 / 2299}$. Equation (68) implies that when $e \sim 1$ the orbital angular momentum is equal to $J=\eta_{t}^{1 / 3} J_{T}$. Since a typical value of $\eta$ allowing survival during the process of tidal circularisation, according to Sect. 5 and Appendix, is slightly larger than the typical value $\eta \approx 4$, we set $\eta_{t}=5$ hereafter. Now we can equate the standard expression $J=$ $\sqrt{G M a\left(1-e^{2}\right)}$ to what is given in Eq. (68), and using Eq. (67) with $\eta_{t}=5$ obtain an implicit equation for the eccentricity $e$

$0.9 \mu^{1 / 3} \lambda^{-1}\left(1-e^{2}\right)=H^{2}(e) e^{12 / 19}$.

From this equation we obtain $e \approx 0.56$ and $e \approx 0.31$ for the high/low density white dwarfs, respectively. The evolution time can be expressed in the form

$T_{\mathrm{ev}}=1.4 F(e) M_{4}^{-2 / 3} P_{2}^{8 / 3} \mathrm{yr}$,

where $P_{2}=P_{\mathrm{orb}} / 100 \mathrm{~s}$ and the function $F(e)$ specifies the dependence of $T_{\mathrm{ev}}$ on $e$, see Peters (1964). We have $F(e=0)=1$, $F(e=0.31) \approx 0.8$ and $F(e=0.56) \approx 0.2$. Note, that an analogous expression for $T_{\mathrm{ev}}$ given in Ivanov (2002) in his Eq. (79) contains an error in last expression on the right hand side and 
must be multiplied on additional factor $M /\left(10^{6} M_{\odot}\right)$. As a result, although his estimate of a probability of finding a source of gravitational waves in galactic centres containing black holes of masses $\sim 10^{6} M_{\odot}$ (Eq. (80) of his paper) is numerically correct for the parameters he uses, it has a different scaling with the black hole mass. It must be multiplied by an additional factor $M /\left(10^{6} M_{\odot}\right)$ which results in smaller values of the probability for black hole masses in the range $\sim 10^{4}-10^{6} M_{\odot}$.

Now, substituting (70) in (66) we obtain minimal values of the amplitude $h, h_{\min }$, which can be detected by LISA

$h_{\min }^{(l)} \approx 10^{-24} M_{4}^{1 / 3}, \quad h_{\min }^{(h)} \approx 5 \times 10^{-24} M_{4}^{1 / 3}$,

where $h_{\min }^{(l, h)}$ stands for the minimal amplitudes for the cases of low/high density white dwarfs, respectively, and we set $P_{2}=1$.

In order to find the total volume of space, available for detection of the signals by LISA, we should relate the quantities $h_{\min }^{(l, h)}$ to the distance from a source, $R_{\text {obs. }}$. For that the amplitude $h$ of radiation emitted by the source should be known as a function of distance and of parameters of the source. The appropriate relation has been obtained, e.g., by Nelemans et al. (2001). In our units it has the form

$h\left(R_{\mathrm{obs}}\right) \approx 5 \times 10^{-21} \mu f(n, e) M_{4}^{2 / 3} P_{2}^{-2 / 3} \frac{1 \mathrm{Mpc}}{R_{\mathrm{obs}}}$,

where the function $f(n, e)$ contains information about the amplitudes associated with harmonics of different order $n$. It is related to the function $g(n, e)$ calculated by Peters \& Mathews (1963) through $f(n, e)=\sqrt{g(n, e)} / n^{5}$. We have $f(n, e=0.56) \approx 0.2$ for several harmonics with $n=1,2,3$ and $f(n=2, e=0.31) \approx 0.4$. Now we can equate $h\left(R_{\mathrm{obs}}\right)$ to $h_{\mathrm{min}}^{(1,2)}$ and find the maximal distance to a source which can be, in principal, detected by LISA

$R_{\mathrm{obs}}^{(l)}=1200 M_{4}^{1 / 3} \mathrm{Mpc}, \quad R_{\mathrm{obs}}^{(h)}=200 M_{4}^{1 / 3} \mathrm{Mpc}$,

where the upper indices $(l, h)$ stand for the cases of low/high density white dwarfs hereafter.

In order to find the total number of potential sources, $N_{\text {tot }}$, we use an estimate obtained by Portegies Zwart \& McMillan (2000) for the average number of globular clusters in the Universe, $n_{\text {G.C. }} \approx 3 \mathrm{Mpc}^{-3}$ for the value of the Hubble constant $H=$ $70 \mathrm{~km} \mathrm{~s}^{-1} \mathrm{Mpc}^{-16}$. We obtain from Eq. (73)

$N_{\text {tot }}^{(l)}=\frac{4 \pi}{3} n_{\text {G.C. }} \Delta\left(R_{\mathrm{obs}}^{(1)}\right)^{3} \approx 2 \times 10^{10} \Delta M_{4}$,

$N_{\mathrm{tot}}^{(h)} \approx 10^{8} \Delta M_{4}$,

where $\Delta$ is the fraction of globular clusters having black holes with masses $\sim M_{4}$. The total probabilities of finding of a source, $\operatorname{Pr}^{(1,2)}$, can be estimated as products of the circularisation rates given by Eqs. (60) and (61) for case a) and Eqs. (64) and (65) for case $b$ ), the total numbers of the sources available and the operational time of the LISA mission taken to be equal to $2 \mathrm{yr}$. have

For case a) with $t_{n l} \approx t_{\mathrm{ev}}$, using Eqs. (60), (61) and (74) we

$\operatorname{Pr}^{(l)} \approx 30 \alpha, \quad \operatorname{Pr}^{(h)} \approx 0.4 \alpha$,

5 Note a misprint in Appendix of Peters \& Mathews (1963) in their expression for $g(n, e)$. The fifth term in the braces must contain the factor $(4 / e)^{2}$ contrary to the factor $\left(4 / e^{2}\right)^{2}$ as in the text.

6 Note that the average number of globular clusters is meaningful only when $R_{\mathrm{obs}}>70 \mathrm{Mpc}$, where $70 \mathrm{Mpc}$ is a typical distance between the elements of the Large Scale Structure in the Universe. where

$\alpha=\Delta \delta_{0.1} M_{4}^{1.57} r_{0.1}^{-1.5}$

It is seen from Eq. (75) that the high density white dwarfs of Sirius B type give rather small probability of detection. On the other hand, the "ordinary" white dwarfs could provide, in principal, a source of gravitational waves provided that our criterion of white dwarfs survival is valid and that

$\alpha>1 / 30$.

It is interesting reformulate the criterion (77) in term of a typical velocity of stars near the radius of influence of the black hole, $\sigma=\sqrt{G M / r_{a}}$. We have from Eqs. (76) and (77)

$\Delta>\frac{1}{30 \delta_{0.1}}\left(\frac{20 \mathrm{~km} \mathrm{~s}^{-1}}{\sigma}\right)^{3}$,

where we neglect a very slow dependence on the black hole mass, $\alpha(\sigma, M) \propto M^{0.07}$. Of course, the black hole mass must be sufficiently large for the validity of the assumptions leading to Eq. (77).

For case b) with $t_{n l} \ll t_{\mathrm{ev}}, t_{\mathrm{dec}}$ we proceed in a similar manner but use Eqs. (64) and (65) instead of Eqs. (60) and (61), respectively. We find

$\operatorname{Pr}^{(l)} \approx 70 \alpha, \quad \operatorname{Pr}^{(h)} \approx 1.5 \alpha$

where

$\alpha=\Delta \delta_{0.1} M_{4}^{2.2} r_{0.1}^{-1.5}$

and

$\Delta>\frac{1}{70 \delta_{0.1}}\left(\frac{20 \mathrm{~km} \mathrm{~s}^{-1}}{\sigma}\right)^{3} M_{4}^{-0.7}$,

instead of Eq. (78) for a "typical low density" white dwarf.

As for case a) "typical" white dwarfs have a much larger probability of detection. However, for case b) this probability depends explicitly on the black hole mass when the cusp size is expresses in terms of the typical stellar velocity dispersion $\sigma$. As follows from Eqs. (79)-(81) when $M_{4} \sim 1$ and $\sigma \sim 20 \mathrm{~km} \mathrm{~s}^{-1}$ this probability is about two times larger than that corresponding to case a). But the probability ratio decreases with the black hole mass if $\sigma$ is kept fixed.

\section{Discussion}

In this paper we have given a qualitative analysis of the problem of tidal circularisation of white dwarfs in globular clusters containing black holes with masses in the range $10^{3}-10^{4} M_{\odot}$ and estimated the rate of production of white dwarfs that begin to circularise their orbits for specified parameters for the cluster, white dwarf and black hole, see Eq. (53). We also proposed a simple criterion for "survival" of a white dwarf during the stage of orbital circularisation, and found production rates for white dwarfs, which can, in principal, settle down in a quasicircular orbit thus forming sources of gravitational radiation, see Eqs. (59-61) and (63-65). These rates are order of magnitude smaller than that given by Eq. (53). We made a simple estimate of the probability of detection of systems containing white dwarfs on quasi-circular orbits and found that these systems can, in principle, be detected by LISA provided that the globular clusters containing black holes are sufficiently abundant 
and their stellar velocity dispersion near the radius of influence of the black hole is sufficiently large, see Eqs. (78) and (81).

Our results should be treated with caution. Many processes occurring during the formation of circularising stars and during ongoing tidal circularisation depend strongly on the parameters of the problem and this leads to uncertainties in the results. Let us consider some of them.

1) In a quantitative approach a realistic model of a white dwarf with non-zero temperature should be used. The eigen spectrum of perturbations should include the gravity/inertial modes. The realistic model should also take into account different channels of mode decay and a self-consistent change of structure of the white dwarf as a result of radiative cooling/heating due to dissipation of the mode energy.

2) Our results indicate that the stars can achieve very high rotation rates during the process of ongoing tidal circularisation. The possibility of survival of white dwarfs during this stage critically depends on tidal exchange of energy between the orbit and the modes of a fast rotating star. It will be very important to generalise well known results of the theory of dynamic tides in non-rotating/slowly rotating stars to the case of fast rotators.

3) As follows from this discussion, our results depend on the rate of mode energy and angular momentum transfer to the star. Possible channels for this transfer, especially through dissipation resulting from non linear effects should be further investigated.

4) The dependence of the circularisation rate on the cusp size and black hole mass should be checked with numerical Nbody simulations. Also, a more realistic distribution of the white dwarfs over the cluster as well as a change of their relative fraction due to stellar evolution should be accounted for in a numerical study.

5) We have adopted a rather simplistic criterion for detection of the gravitational waves produced by the white dwarf orbiting close to a black hole, assuming that the amplitude of the signal is equal to the noise amplitude of the detector. Higher $\mathrm{S} / \mathrm{N}$ detection threshold would result in a much smaller probability of detection. On the other hand, a more sophisticated strategy of signal processing could improve our estimates.

We have shown that during the first stage of orbital circularisation the orbital energy transferred to the fundamental mode of stellar pulsations may be radiated away by gravitational waves. That means that the globular clusters may contain sources of gravitational radiation with a typical frequency of the order of the eigen frequency of the fundamental quadrupole mode, of the order of a few Hz. The possibility of detection of such sources needs further investigation.

Finally we comment that in a recent paper Baumgardt et al. (2006) have calculated the tidal capture rates for stars of different types with help of $N$-body simulations, during first $12 \mathrm{Myrs}$ of evolution of the system. They have considered black holes with masses in the range $\sim 10^{3}-4 \times 10^{3} M_{\odot}$. Giant stars and low mass main sequence stars with mass $<0.4 M_{\odot}$ have been modelled as $n=1.5$ polytropes. The low mass stars seem to be absent in the cusp due to the effect of mass segregation operating during formation of the cusp. However, their capture rate of red giants can be compared with what is given by Eq. (53). They have obtained a capture rate for red giants of the order of a few events per run. Assuming that a typical red giant has a radius $\sim 10^{3} R_{\odot}$, mass $\sim 10 M_{\odot}$, taking the black hole mass and the cusp size to be equal to $3 \times 10^{3} M_{\odot}$ and to $0.1 \mathrm{pc}$, respectively, and substituting these values into Eq. (53), we obtain $\dot{N}_{\text {R.G. }} \sim 3 \times 10^{-5} \delta_{\text {R.G. }} \mathrm{yr}^{-1}$, where $\delta_{\text {R.G. }}$ is the number fraction of the red giants in the cusp. Our results appears to be in full agreement with the results of $N$-body simulations provided that $\delta_{\text {R.G. }} \sim 1 / 100$.

Acknowledgements. We are grateful to A. G. Polnarev for fruitful discussions to A. G. Doroshkevich for useful remarks and to the referee for valuable comments. P.B.I. has been supported in part by RFBR grants 04-02-17444 and 07-02-00886.

\section{Appendix A: Conditions for the transition from stochastic instability}

As indicated in Sect. 3.7, there is a phase of evolution at large semi-major axis subsequent to tidal capture where the internal pulsation mode energy may increase in a stochastic manner. This requires the semi-major axis to exceed $a_{\text {st }}$ above which the pulsation mode does not maintain phase coherence between successive pericentre passages.

Here we obtain an explicit expression for the semi-major axis $a_{\text {st }}$ defined through Eq. (13). At first let us assume that $\Delta E_{T}>\Delta E_{G W}$ during the orbital evolution. This situation is illustrated in Figure 2. In this case we substitute Eq. (7) in Eq. (13) and obtain

$a_{\mathrm{st}} \equiv a_{\mathrm{st}}^{(1)} \approx 1.15 \times 10^{11} \lambda \mu^{-3 / 5} M_{4}^{3 / 5}(\phi \Psi)^{2 / 5} \mathrm{~cm}$.

Now let us consider the orbital evolution when $\Delta E_{T}<\Delta E_{G W}$. This situation is illustrated in Figure 1. In this case the semimajor corresponding to the onset of the stochastic instability, $a_{\mathrm{st}} \equiv a_{\mathrm{st}}^{(2)}$ can be obtained by substitution of Eq. (28) in Eq. (13) with the result

$a_{\mathrm{st}}^{(2)} \approx 1.7 \times 10^{12} \eta^{14 / 15} \lambda^{2} \mu^{-16 / 15} M_{4}^{1 / 15} \mathrm{~cm}$.

Just after tidal capture occurs the orbital evolution is primarily due to tides. The orbit decays until $a_{\mathrm{st}}$ is reached but at this point it is possible that the orbital evolution is governed by gravitational radiation. As indicated in Sect. 5, when the orbital semimajor axis is of the order of $a_{\mathrm{st}}$ there are three non trivial possibilities: case a1) where $t_{n l} \approx t_{\mathrm{ev}}$ and tides determine the orbital evolution; case a2) where $t_{n l} \approx t_{\mathrm{ev}}$ and gravitational radiation determines the orbital evolution; and case b2) where $t_{n l} \ll t_{\mathrm{ev}}$ and gravitational radiation controls the orbital evolution. Each of these is considered in more detail below.

\section{A.1. The case a1: tides dominate and $t_{\mathrm{nl}}$ is large}

As indicated in Sects. 4 and 5, when $t_{n l} \sim t_{\mathrm{ev}}$ a white dwarf has a possibility of surviving the process of tidal circularisation only if it is cooled efficiently by emission of gravitational waves for values of its semi-major axes $>a_{\mathrm{st}}^{(1)}$. Therefore, in order to reach a quasi-circular orbit, the white dwarf must have orbital parameters such that $a_{\mathrm{st}}^{(1)}>a_{\mathrm{dis}}$ where $a_{\mathrm{dis}}$ is given by Eq. (32). This inequality leads to an inequality of the form $\eta>\eta_{1}$ (or equivalently the fixed pericentre distance must exceed a certain value), where $\eta_{1}$ is defined by the condition $a_{\mathrm{st}}^{(1)}=a_{\mathrm{dis}}$. In general, $\eta_{1}$ is a monotonic function of the stellar rotation rate with smaller values of $\eta_{1}$ corresponding to larger values of $\Omega_{r}$. This is because tides weaken with increasing $\Omega_{r}$ enabling safe circularisation starting with smaller pericentre distances.

Thus in order to take into account all possible evolutionary tracks leading to formation of circular orbits, we calculate the 
lower boundary of $\eta_{1}$ assuming that $\Omega_{r}=\Omega_{\mathrm{br}} \approx 0.5 \Omega_{*}$. In this case the factor $(\phi \Psi)$ entering (A.1) takes the form

$(\phi \Psi) \approx \frac{1}{\eta} \exp (3.68 \eta-2.74)$

Substituting Eq. (A.3) in Eq. (A.1) and Eqs. (32) and (A.1), we obtain

$\eta_{1} \approx 4+0.27\left(\ln \eta_{1}+\ln Z_{1}\right)$

where $Z_{1}=\lambda^{25 / 16} \mu^{-21 / 16} M_{4}^{-1 / 4}$.

In the subsequent discussion we use the quantity $\phi_{1} \equiv \phi\left(\eta_{1}\right)$ defined in Eq. (8) rather than $\eta_{1}$. This quantity can be approximately represented in the form

$\phi_{1} \approx 2.4 \times 10^{3} Z_{1}^{0.7} \approx 2.4 \times 10^{3} \lambda^{1.1} \mu^{-0.92} M_{4}^{-0.175}$,

with the difference between this power-law fit and the solution of Eq. (A.4) being less than or of the order of 5 per cent for all interesting values of $Z_{1}$.

\section{A.2. The case a2: gravitational waves dominate and $t_{n l}$ is large}

Now let us assume that gravitational waves determine the orbital evolution when a rapidly rotating white dwarf has its semi-major axis near to $a_{\mathrm{dis}}$ and $t_{n l} \approx t_{\mathrm{ev}} \approx t_{G W}$, see Fig. 1. Proceeding as in the preceding section, we obtain an equation for the critical value of $\eta_{2}$ that is analogous to Eq. (A.4) in the form

$\eta_{2} \approx 4.88-0.11 \ln \eta_{2}+0.27 \ln Z_{2}$,

where $Z_{2}=\lambda \mu^{-7 / 5} M_{4}^{2 / 5}$. The corresponding value of $\phi_{2} \equiv \phi\left(\eta_{2}\right)$ can also be, with a high accuracy, represented in the power-law form

$\phi_{2} \approx 5.4 \times 10^{3} Z_{2}^{13 / 20} \approx 5.4 \times 10^{3} \lambda^{0.65} \mu^{-0.91} M_{4}^{0.26}$.

The value of the parameter $\eta$ and thus $\phi(\eta)$ which divides, according to our criterion, orbits leading to a safe circularisation from orbits leading to a possible disruption of the white dwarf, is obtained from the condition $\phi(\eta)>\max \left(\phi_{1}, \phi_{2}\right)$. We note that the condition for gravitational radiation to dominate, $\phi_{2}>\phi_{1}$, leads to the approximate condition on the black hole mass that

$M_{4}>M_{*}=0.155 \lambda$,

where we have neglected a very weak dependence of $M_{*}$ on $\mu$ of the form $M_{*} \propto \mu^{-0.02}$, the latter quantity being replaced by unity.

The inequality (A.8) implies that for a sufficiently large values of the black hole mass and the rotation of the white dwarf the orbital evolution is mainly determined by emission of gravitational waves when the semi-major of the orbit is close to the characteristic semi-major axis corresponding to the onset of stochastic instability. For the parameters corresponding to a "typical" white dwarf with $\mu=0.6$ and $\lambda=1.4$, we have $M_{*} \approx 0.22$, and for a dense white dwarf of Sirius B type with $\mu=1$ and $\lambda=0.84$, we have $M_{*}=0.13$. Both characteristic values of $M_{*}$ are smaller than the black hole masses of systems expected to produce a large amount of gravitational radiation during the last stages of orbital evolution of the star. Therefore, the condition $\eta>\eta_{2}$ is more important for our purposes.

\section{A.3. The case b2: gravitational waves dominate and $t_{\mathrm{nl}}$ is small}

Now let us consider the case when $t_{n l} \ll t_{\mathrm{ev}}, t_{\mathrm{dec}}$. The discussion proceeds in a similar way to the previous case but now $a_{\mathrm{dis}}$ is given by Eq. (31). As we have mentioned above it can then be shown that gravitational waves dominate the orbital evolution when the semi-major axis is $\sim a_{\text {dis }}$ provided that the black hole mass exceeds $\sim 10^{3} M_{\odot}$, and accordingly, $t_{\mathrm{ev}} \approx t_{G W}$.

Therefore in order to obtain an equation for the parameter $\eta \equiv \eta_{3}$ separating orbits leading to safe circularisation from those leading to disruption of the star, we equate expressions (31) and (A.2), and use Eqs. (7), (28), (29) and (A.3), to obtain

$\eta_{3}=3.5+0.65 \ln \eta_{3}+0.27 \ln Z_{3}$,

where $Z_{3}=\lambda^{3 / 2} \mu^{-6 / 5} M_{4}^{-8 / 15}$. An approximate solution to this equation can be found from

$\phi_{3} \equiv \phi\left(\eta_{3}\right) \approx 2 \times 10^{3} \lambda^{6 / 5} \mu^{-24 / 25} M_{4}^{-32 / 75}$.

\section{References}

Bahcall, J. N., \& Wolf, R. A. 1976, ApJ, 209, 214

Baumgardt, H., Makino, J., \& Ebisuzaki, T. 2004a, ApJ, 613, 1133

Baumgardt, H., Makino, J., \& Ebisuzaki, T. 2004b, ApJ, 613, 1144

Baumgardt, H., Hopman, C., Portegies Zwart, S. F., \& Makino, J. 2006, MNRAS, 372, 467

Chugunov, A. I., \& Yakovlev, D. G. 2005, Astron. Rep., 49, 724

Clement, M. J. 1989, ApJ, 339, 1022

Fabbiano, G. 1989, A\&ARv, 27, 87

Frank, J., \& Rees, M. J. 1976 MNRAS, 176, 633

Freitag, M. 2000, ApJ, L21

Frolov, V. P., Khokhlov, A. M., Novikov, I. D., \& Pethick, C. J. 1994, ApJ, 432, 680

Gebhardt, K., Pryor, C., O’Connel, R. D., Williams, T. B., \& Hesser, J. E. 2000, AJ, 119,1268

Gebhardt, K., Rich, R. M., \& Ho, L. C. 2002, ApJ, 578, L41

Ghosh, K. K., Suleymanov, V., Bikmaev, I., Shimansky, S., \& Sakhibullin, N. 2006, MNRAS, 371, 1587

Goldreich, P., \& Nicholson, P. D. 1989, ApJ, 342, 1075

Grishchuck, L. P., Lipunov, V. M., Postnov, K. A., Prokhorov, M. E., \& Sathyaprakash, B. S. 2001, Physics-Uspekhi, 44, 1

Gurevich, A. V. 1964, Geomagnetism and Aeronomy, 4, 192

Hopman, C., \& Alexander, T. 2005, ApJ, 629, 362

Ivanov, P. B. 2002, MNRAS, 336, 373

Ivanov, P. B., \& Novikov, I. D. 2001, ApJ, 549, 467

Ivanov, P. B., \& Papaloizou, J. C. B. 2004, MNRAS, 347, 437

Kochanek, C. S. 1992, ApJ, 385, 604

Kosovichev, A. G., \& Novikov, I. D. 1992, MNRAS, 258, 715

Lai, D. 1997, ApJ, 490, 847

Lightman, A. P., \& Shapiro, S. L. 1977, ApJ, 211, 244

Lee, H. M., \& Ostriker, J. B. 1966, ApJ, 310, 176

Managan, R. A. 1986, ApJ, 309, 598

Mardling, R. A. 1995, ApJ, 450, 722

Matsumoto, H., Tsuru, T. G., \& Koyama, K. 2001, ApJ, 547, L25

Miller, C. M., \& Hamilton, D. P. 2002, MNRAS, 330, 232

Nelemans, G., Yungelson, L. R., \& Portegies Zwart, S. F. 2001, A\&A, 375, 890

Novikov, I. D., Pethick, C. J., \& Polnarev, A. G. 1992, MNRAS, 225, 276

Osaki, Y., \& Hansen, C. J. 1973, ApJ, 277

Papaloizou, J. C. B., \& Ivanov, P. B. 2005, MNRAS, 364, L66

Peters, P. C. 1964, Phys. Rev., 136, 1224

Peters, P. C., \& Mathews, J. 1963, Phys. Rev., 131, 435

Portegies Zwart, S. F., \& Mc Milllan, S. L. W. 2000, ApJ, 528, L17

Press, W. H., \& Teukolsky, S. A. 1977, ApJ, 213, 183

van der Marel, R. P. 2004, in Coevolution of Black Holes and Galaxies, from Carnegie Observatories Centennial Symposia (Cambridge University Press as Part of the Carnegie Observatories Astrophysics Series), ed. L. C. Ho, 37 Young, P. 1980, ApJ, 242, 1232 